\title{
Conformal and Affine Hamiltonian Dynamics of General Relativity
}

\author{
Victor N. Pervushin - Andrej B. \\ Arbuzov • Boris M. Barbashov • Rashid \\ G. Nazmitdinov • Andrzej Borowiec . \\ Konstantin N. Pichugin • Alexander F. \\ Zakharov
}

Received: date / Accepted: date

\begin{abstract}
The Hamiltonian approach to the General Relativity is formulated as a joint nonlinear realization of conformal and affine symmetries by means of the Dirac scalar dilaton and the Maurer-Cartan forms. The dominance of the Casimir vacuum energy of physical fields provides a good description of the type Ia supernova luminosity distance-redshift relation. Introducing the uncertainty principle at the Planck's epoch within our model, we obtain the hierarchy of the Universe energy scales, which is supported by the observational data. We found that the invariance of the Maurer-Cartan forms with respect to the general coordinate transformation yields a single-component strong gravitational waves. The Hamiltonian dynamics of the model describes the effect of an intensive vacuum creation of gravitons and the minimal coupling scalar (Higgs) bosons in the Early Universe.
\end{abstract}

Keywords General Relativity, Conformal Cosmology

V.N. Pervushin, A.B. Arbuzov, B.M. Barbashov, R.G. Nazmitdinov, A.F. Zakharov

Bogoliubov Laboratory of Theoretical Physics, Joint Institute for Nuclear Research, 141980 Dubna, Russia

Tel.: +7-49621-63343

E-mail: arbuzov@theor.jinr.ru

A.B. Arbuzov

Department of Higher Mathematics, University of Dubna, 141980 Dubna, Russia

R.G. Nazmitdinov

Department de Física, Universitat de les Illes Balears, E-07122 Palma de Mallorca, Spain

A. Borowiec

Institute of Theoretical Physics, University of Wrocław, Pl. Maxa Borna 9, 50-204 Wrocław, Poland

K.N. Pichugin

Kirensky Institute of Physics, 660036 Krasnoyarsk, Russia

A.F. Zakharov

Institute of Theoretical and Experimental Physics, B. Cheremushkinskaya str. 25, 117259

Moscow, Russia 
PACS 95.30.Sf, 98.80.-k, 98.80.Es

\section{Introduction}

The Standard Model (SM) of electroweak and strong interactions 1 describes well practically all physical phenomena up to the energy of at least $100 \mathrm{GeV}$. According to the accepted wisdom, the physical content of the SM (in the lowest order of perturbation theory) is completely covered by fields and particles as representations of the Poincaré group (with positively defined Hilbert space scalar product) and the Lorentz classification of variables used for calculation of weak transitions between these states [2].

The unification of the SM with a gravitational theory is a longstanding fundamental problem. It seems natural in this case to require that both theories should be treated on equal footing. The main difficulty of the unification lies in the different theoretical levels of their presentation: quantum for the SM and classical for the gravitational theory. However, both these theories have common roots of their origin (mechanics and electrodynamics) and obey the principles of relativity confirmed by numerous experimental observations. Note that there is also enormous progress in observational cosmology 3. 4 which enters into the era of precise science; it means that a typical accuracy of standard parameter determination is about a few per cent. Evidently, one of the major aims of the unification is to develop a cosmological model which could pass the vitality test by the cosmological data. Last but not least, this model should allow one to develop a renormalizable quantized version.

The first step in this direction is due to Fock 5. He introduced the Dirac electron as a spinor representation of the Lorentz group into the General Relativity (GR) by means of the Einstein interval as the sum of squared linear forms. These forms are known as four components of a simplex of the reference frame in the tangent Minkowskian space-time. The next step was made in [6, where it was shown that infinite-parametric general coordinate transformations introduced by Einstein 7] can be converted to the finite-parametric conformal group and the affine group of all linear transformations of fourdimensional space-time, including the Poincaré group. The conformal symmetry as a basis for the construction of the GR was independently introduced by Deser and Dirac [8, 9]. In particular, Dirac formulated the conformal-invariant approach to the GR [9] as a new variational principle for the Hilbert action [10. introducing a dilaton (scalar) field, in addition to $g_{\mu \nu}$. Further it was shown [1] that in the case of the dynamical affine symmetries the method of nonlinear realization of symmetry groups 12 leads to the Hilbert action of Einstein's gravitational theory expressed in terms of the Fock simplex components.

A particular conformal cosmological model, based on the ideas discussed above, have been developed in papers 13, 14, 15, 16. It was shown that the model leads to a viable cosmology being in agreement with observations. For example, a good description of the modern supernovae type Ia data was constructed [13, 14] in the assumption of a rigid state dominance. The initial data 
conditions on the dilaton and an additional scalar field have been employed as a source of the conformal symmetry breaking. This field led to the rigid state dominance at the classical level. In the present paper we shall attempt to go beyond the classical level and show that the Casimir vacuum effect in a finitesize Universe could provide both the scale invariance breaking and the rigid state dominance, required in our model to describe the SNe Ia data. Therefore we substantially change the basis of the whole model. For this reason we reformulated below the model and re-derived its phenomenological consequences. Note that recently different approaches to construction of conformal-invariant models of gravity and cosmology have been suggested in the literature, see e.g. papers [17, 18, 19] and references therein.

The goal objective of our paper is to construct a self-consistent gravitational model of the Universe based on the affine and conformal symmetries in the framework of the Dirac variational principle. Our approach enables us: i) to develop the Hamiltonian description of the cosmological evolution, ii) to obtain exact solution of constraints, and iii) using this solution to gain cosmological quantum effects, including the vacuum creation processes at the Planck epoch. The content of the paper is as follows. Section 2 is devoted to the Hamiltonian approach to our gravitation model, clearing up its symplectic structure. Here we also establish the Planck epoch hierarchy of the Universe energy scales defined by the Casimir vacuum energy and the quantum uncertainty principle. In Section 3, we analyze the properties of strong affine gravitational waves in a dynamical approximation, when all static Newton-like potentials are neglected. The conformal cosmological (CC) model is briefly discussed and an intensive vacuum creation of gravitons is described. Section 4 describes gravitational interactions of fermions. In Section 5 we compare the vacuum creation of gravitons with the Higgs particle one. The main results are summarized in Section 6. In Appendix A, we briefly reconsider the Dirac Hamiltonian approach in infinite space-time, reformulated in terms of the Maurer-Cartan forms in order to compare it with our construction in Section 2, In Appendix B we present the dilaton cosmological perturbation theory. Appendix $\mathrm{C}$ is devoted to the conformal cosmology.

\section{Conformal Hamiltonian dynamics}

\subsection{Nonlinear realization of affine and conformal symmetries}

Let us define a conformal version of the GR as a nonlinear realization of joint conformal and affine $A(4)$ symmetries in the factor space $A(4) / L$ with the Lorentz subgroup $L$ of the stable vacuum (here we use the concepts of the theory [12]). Recall that the affine group $A(4)$ is the group of all linear transformations of the four-dimensional manifold $x^{\mu} \rightarrow \widetilde{x}^{\mu}=x^{\mu}+y^{\mu}+L_{[\mu \nu]} x^{\nu}+R_{\{\mu \nu\}} x^{\nu}$, where $y^{\mu}$ is a shift of coordinate and $L_{[\mu \nu]}$ and $R_{\{\mu \nu\}}$ are antisymmetric and symmetric matrices respectively (here Greek indices $\mu, \nu, \ldots$ run from 0 to 3 ). A nonlinear realization of $A(4)$ is based on finite transformations $G=e^{i P \cdot x} e^{i R \cdot h}$ 
defined by means of the shift operator $P$, proper affine transformation $R$ and the following Goldstone modes: four coordinates $x_{\mu}$ and ten gravitational fields $h$ [6]. This realization can be obtained with the aid of the Maurer-Cartan forms 1 in the following way

$$
\begin{aligned}
G d G^{-1} & =i[\underbrace{P_{(\alpha)} \cdot \omega_{(\alpha)}^{P}+R_{(\alpha)(\beta)} \cdot \omega_{(\alpha)(\beta)}^{R}}_{\text {shifts of simplex in } K=A(4) / L}+\underbrace{L_{(\alpha)(\beta)} \cdot \omega_{(\alpha)(\beta)}^{L}}_{\text {rotations in } K=A(4) / L}], \\
\omega_{(\alpha)}^{P}(d) & =e_{(\alpha) \mu} d x^{\mu}, \\
\omega_{(\alpha)(\beta)}^{R}(d) & =\frac{1}{2}\left(e_{(\alpha)}^{\mu} d e_{(\beta) \mu}+e_{(\beta)}^{\mu} d e_{(\alpha) \mu}\right), \\
\omega_{(\alpha)(\beta)}^{L}(d) & =\frac{1}{2}\left(e_{(\alpha)}^{\mu} d e_{(\beta) \mu}-e_{(\beta)}^{\mu} d e_{(\alpha) \mu}\right) .
\end{aligned}
$$

Here, $\omega_{(\alpha)}^{P}(d), \omega_{(\alpha)(\beta)}^{R}(d)$ are shifts of a simplex of the reference frame in the coset space $A(4) / L$, and $\omega_{(\alpha)(\beta)}^{L}(d)$ is responsible for the rotation of the simplex. The explicit dependence of the decomposition coefficient $e_{(\alpha)}^{\mu}, e_{(\beta) \mu}$ (known as tetrades [5]) on the gravitational fields $h$ was obtained in Refs. [11, 21. Note that there are two types of indices: one belongs to the subgroup $L$ and the other (bracket Greek indices $(\alpha),(\beta), \ldots$ run from 0 to 3 ) to the coset $A(4) / L$. In this approach, the Maurer-Cartan forms with the coset indices are main objects of the Poincaré transformations and classification of states. According to the general theory of non-linear realizations [12] we should express all measurable quantities via the coset variables with bracket-indices.

To construct a GR model in this approach, one needs to consider the covariant differentiation of a set of fields $\Psi$ transformed by means of the Lorentz group generators $L_{(\alpha)(\beta)}^{\Psi}$

$$
D_{(\gamma)} \Psi=\frac{D \Psi}{\omega_{(\gamma)}^{P}}=\left[\partial_{(\gamma)}+\frac{1}{2} i v_{(\alpha)(\beta),(\gamma)} L_{(\alpha)(\beta)}^{\Psi}\right] \Psi,
$$

where $\partial_{(\gamma)}=\left(e^{-1}\right)_{\mu(\gamma)} \partial_{\mu}$. Here, the linear form $v_{(\alpha)(\beta),(\gamma)}$ is constructed by means of the Maurer-Cartan forms (3) (4),

$$
v_{(\alpha)(\beta),(\gamma)}=\left[\omega_{(\alpha)(\beta)}^{L}\left(\partial_{(\gamma)}\right)+\omega_{(\alpha)(\gamma)}^{R}\left(\partial_{(\beta)}\right)-\omega_{(\beta)(\gamma)}^{R}\left(\partial_{(\alpha)}\right)\right] .
$$

Similarly, the covariant expression for the action of the Goldstone fields $h$ can be obtained with the aid of the commutator of the covariant differentiation of a set of the fields $\Psi$ 22

$$
\left[D_{(\delta)} D_{(\gamma)}-D_{(\gamma)} D_{(\delta)}\right] \Psi=i R_{(\alpha)(\beta),(\delta)(\gamma)}^{(4)} L_{(\alpha)(\beta)}^{\Psi} \Psi / 2 .
$$

The Riemann curvature tensor is defined as [1]:

$$
\begin{aligned}
& R_{(\alpha)(\beta),(\gamma)(\delta)}^{(4)}=\partial_{(\gamma)} v_{(\alpha)(\beta),(\delta)}+v_{(\alpha)(\beta),(\zeta)} v_{(\delta)(\zeta),(\gamma)} \\
& \quad+v_{(\alpha)(\zeta),(\delta)} v_{(\beta)(\zeta),(\gamma)}-((\gamma) \leftrightarrow(\delta))
\end{aligned}
$$

\footnotetext{
1 These forms were introduced in the GR by Fock and Cartan [5 20]).
} 
The joint conformal realization of the affine and conformal groups $A(4) \times C$ symmetry allows to separate the dilaton field D [9] as the Goldstone mode accompanying the spontaneous conformal symmetry breaking via a scale transformation:

$$
e_{(\alpha)}^{\mu}=\widetilde{e}_{(\alpha)}^{\mu} e^{D}
$$

Using the correspondence principle to the classical gravitation theory and the minimal derivative number postulates, we obtain the conformal-invariant action:

$$
\begin{aligned}
W_{\mathrm{C}}\left[D, \widetilde{e}_{(\alpha) \nu}\right] & =-M_{\mathrm{C}}^{2} \frac{3}{8 \pi} \int d^{4} x\left[\frac{\sqrt{-\widetilde{g}}}{6} R^{(4)}(\widetilde{g}) e^{-2 D}\right. \\
& \left.-e^{-D} \partial_{\mu}\left(\sqrt{-\widetilde{g}} \widetilde{g}^{\mu \nu} \partial_{\nu} e^{-D}\right)\right],
\end{aligned}
$$

where $M_{C}$ is the conformal Newton coupling constant.

In this case the measurable interval $\widetilde{d s}^{2}$ is determined by the conformal metric $\widetilde{g}_{\mu \nu}$ expressed via the tetrades $\widetilde{e}_{(\alpha) \mu}$ :

$$
\widetilde{g}_{\mu \nu}=\widetilde{e}_{(\alpha) \mu} \otimes \widetilde{e}_{(\alpha) \nu} \rightarrow \widetilde{d s}^{2}=\widetilde{g}_{\mu \nu} d x^{\mu} d x^{\nu} .
$$

Note that the standard Hilbert-Einstein action

$$
W_{\mathrm{E}}[g]=-\left(M_{\mathrm{Pl}}^{2} / 16\right) \int d^{4} x \sqrt{-g} R^{(4)}(g)
$$

with the standard Einstein interval

$$
d s_{\mathrm{E}}^{2}=g_{\mu \nu} d x^{\mu} d x^{\nu}
$$

can be reproduced:

$$
W_{\mathrm{C}}\left[D, \widetilde{e}_{(\alpha) \nu}\right]=W_{\mathrm{E}}[g], \quad \text { if } \quad\left\{\begin{array}{l}
g_{\mu \nu}=e^{2 D} \widetilde{e}_{(\alpha) \nu} \otimes \widetilde{e}_{(\alpha) \nu} \\
M_{\mathrm{Pl}}=M_{\mathrm{C}}
\end{array}\right.
$$

In the action (10) we have joined two approaches: the Dirac's dilaton conformal theory and the Fock tetrades with the affine symmetry. Although there is a formal correspondence (14), some physical consequences, as will be shown below, are different. We point out that in our approach there is a new set of dynamical variables $\left\{\widetilde{e}_{(\alpha)}^{\mu}, D\right\}$ which are subject to the affine and conformal symmetry constraints. In particular, the conformal invariant interval $\widetilde{d s}^{2}$ substitutes the standard one $d s_{\mathrm{E}}^{2}$. It will be shown below that the new variables enable to us to find explicit solutions for all symmetry constraints in the framework of the Dirac approach [23]. One of the key assumptions of our approach to the GR is that measurable quantities are identified with the conformal field variables $\widetilde{F}_{(n)}$. These variables are obtained from the standard ones $F_{(n)}$ by means of the Weyl transformation $F_{(n)}=\widetilde{F}_{(n)} e^{n D}$, where $n$ is the conformal 
weight 24. Therefore, we name our approach as a conformal general relativity (CGR) approach to the gravitation theory.

Below we will use the natural units:

$$
M_{\mathrm{Pl}} \sqrt{3 /(8 \pi)}=c=\hbar=1 .
$$

\subsection{Conformal formulation of the Dirac-ADM foliation $4 \rightarrow 1+3$}

Thus, we have defined the action and the variables of our model. In order to obtain physical results we have to resolve within the Hamiltonian approach the constraints arisen due to the affine and conformal symmetries.

Let us reformulate the Dirac-ADM foliation 23,25] in terms of the simplex components and the dilaton 2 . The simplex components $\left[\widetilde{\omega}_{(0)}, \widetilde{\omega}_{(b)}\right]$ (here all Latin indices run from 1 to 3 ) can be written as

$$
\begin{aligned}
& \widetilde{\omega}_{(0)}=e^{-2 D} N d x^{0}, \\
& \widetilde{\omega}_{(b)}=\widetilde{\mathbf{e}}_{(b) i} d x^{i}+N_{(b)} d x^{0},
\end{aligned}
$$

where $N_{(b)}=N^{j} \widetilde{\mathbf{e}}_{j(b)}$ are the shift vector components, and $N\left(x^{0}, x^{j}\right)$ is the lapse function. Here $\widetilde{\omega}_{(b)}$ are the linear forms defined via the triads $\widetilde{\mathbf{e}}_{(b) i}$ with a unit spatial metric determinant

$$
\left|\widetilde{\mathbf{e}}_{j(b)}\right|=1,
$$

i.e., the Lichnerowicz gauge [26]-type for the triads. This gauge connects the scalar dilaton field $D$ with a logarithm of the Einstein metric determinant:

$$
D=-(1 / 6) \ln \left|g_{i j}^{(3)}\right| .
$$

Recall that this component was distinguished by Dirac in his Hamiltonian approach to the Einstein GR 23 .

The group of invariance of the GR for the Dirac-ADM foliation is known as the kinemetric subgroup of the general coordinate transformation [27]

$$
\begin{aligned}
x^{0} \rightarrow \widetilde{x}^{0} & =\widetilde{x}^{0}\left(x^{0}\right), \\
x^{k} \rightarrow \widetilde{x}^{k} & =\widetilde{x}^{k}\left(x^{0}, x^{1}, x^{2}, x^{3}\right) .
\end{aligned}
$$

This group admits the decomposition of the dilaton into the sum of the zeroth and nonzeroth harmonics:

$$
D\left(x^{0}, x^{1}, x^{2}, x^{3}\right)=\langle D\rangle\left(x^{0}\right)+\bar{D}\left(x^{0}, x^{1}, x^{2}, x^{3}\right) .
$$

\footnotetext{
2 Although the Dirac Hamiltonian approach to the Hilbert action in terms of the Dirac$\mathrm{ADM}$ metric components is well known 23,25, for the sake of comparison we present in Appendix A the modification of this approach in terms of the Maurer-Cartan forms.
} 
This is one of the key points in our construction. The introduction of the zeroth mode $\langle D\rangle\left(x^{0}\right)$ is consistent with the Einstein cosmological principle of averaging of all scalar fields of the theory over a finite volume $V_{0}=\int_{V_{0}} d^{3} x[28$.

$$
\langle D\rangle\left(x^{0}\right)=V_{0}^{-1} \int_{V_{0}} d^{3} x D\left(x^{0}, x^{1}, x^{2}, x^{3}\right) .
$$

In virtue of Eqs. (22) and (23), we obtain the orthogonality condition

$$
\int_{V_{0}} d^{3} x \bar{D}\left(x^{0}, x^{1}, x^{2}, x^{3}\right) \equiv 0 .
$$

This condition enables to us to consider the zeroth and nonzeroth components as independent ones.

The invariance of the action with respect to the reparameterization of the coordinate time parameter (20) guides us to suppose that the zeroth dilaton mode $\langle D\rangle\left(x^{0}\right)$ can be chosen as an evolution parameter in the field space of events $\left[\langle D\rangle, \bar{D}, \mathbf{e}_{(b)}^{j}\right][15]$. Note that by definition the zeroth dilaton harmonics (obtained by averaging it over a finite volume) coincides with the cosmological scale factor logarithm 29

$$
\langle D\rangle=-\ln a=\ln (1+z) .
$$

The factorization of the lapse function

$$
N\left(x^{0}, x^{j}\right)=N_{0}\left(x^{0}\right) \mathcal{N}\left(x^{0}, x^{j}\right)
$$

by the spatial volume average 15

$$
\left\langle N^{-1}\right\rangle \equiv \frac{1}{V_{0}} \int_{V_{0}} d^{3} x \frac{1}{N\left(x^{0}, x^{1}, x^{2}, x^{3}\right)}=N_{0}^{-1}\left(x^{0}\right) .
$$

yields the diffeo-invariant proper dilaton time interval $d \tau$ connected with the world time interval $d t$ and the conformal one $d \eta$ as

$$
d \tau=N_{0}\left(x^{0}\right) d x^{0}=a^{-2} d \eta=a^{-3} d t .
$$

Here, we used the obvious normalization condition for the diffeo-invariant lapse function

$$
\left\langle\mathcal{N}^{-1}\right\rangle \equiv \frac{1}{V_{0}} \int_{V_{0}} d^{3} x \frac{1}{\mathcal{N}\left(x^{0}, x^{j}\right)}=1 .
$$

This classification of time-intervals (dilaton, conformal, and world ones) enables one to introduce the corresponding Hubble parameters

$$
\begin{aligned}
H_{\tau} & \equiv-\partial_{\tau}\langle D\rangle, \\
H_{\eta} & \equiv-\partial_{\eta}\langle D\rangle, \\
H_{t} & \equiv-\partial_{t}\langle D\rangle .
\end{aligned}
$$


The choice of the zeroth dilaton mode $\langle D\rangle$ as an evolution parameter has two consequences in the Hamiltonian approach. First, the zeroth dilaton mode canonical momentum density

$$
P_{\langle D\rangle}=\frac{2}{V_{0}} \int_{V_{0}} d^{3} x \sqrt{-g} g^{00} \frac{d}{d x^{0}}\langle D\rangle \equiv 2 \frac{d}{d \tau}\langle D\rangle=2 v_{\langle D\rangle}=\text { Const. } \neq 0
$$

can be treated as a generator of the Hamiltonian evolution in the field space of events 30,31 . Here $v_{\langle D\rangle}$ is the corresponding velocity, by construction it coincides with $H_{\tau}$ introduced in Eq. (30). We stress that the scale-invariance $(D \rightarrow D+\Omega)$ admits only a constant $P_{\langle D\rangle}$.

The second consequence of the orthogonality condition (24) is that the nonzeroth harmonics $\bar{D}\left(x^{0}, x^{1}, x^{2}, x^{3}\right)$ do not depend on the evolution parameter. Therefore, the canonical momentum of dilaton nonzeroth modes is equal to zero:

$$
P_{\bar{D}} / 2=v_{\bar{D}}=\left[\left(\partial_{0}-N^{l} \partial_{l}\right) \bar{D}+\partial_{l} N^{l} / 3\right] / N=0 .
$$

Note that in the Dirac approach, the condition $v_{\bar{D}}=0$ was also introduced as an additional second class constraint [23,33, see Appendix A. In this case the nonzeroth modes play the role of gravitational Newton-type potentials as the lapse function and the shift vector do.

This result fixes the longitudinal shift vector component A.24). As a result, we have

$$
\int d^{3} x v_{\langle D\rangle} \cdot v_{\bar{D}}=0,
$$

that follows from of Eqs. (22), (23), and (24). The orthogonality conditions (24) and (35) preserve the definite metrics in the Hilbert space of states 2, 32 .

Thus taking into account Eqs. (22) and (25)), we have the following action:

$$
\begin{gathered}
W_{\mathrm{C}}=\underbrace{W_{\text {Universe }}}_{=0 \text { for } \mathrm{V}_{0}=\infty}+W_{\text {graviton }}+W_{\text {potential }}, \\
W_{\text {Universe }}=-V_{0} \int_{\tau_{I}}^{\tau_{0}} \underbrace{d x^{0} N_{0}}_{=d \tau}\left[\left(\frac{d\langle D\rangle}{N_{0} d x^{0}}\right)^{2}+\rho_{\tau}^{\mathrm{v}}\right], \\
W_{\text {graviton }}=\int d^{4} x \frac{N}{6}\left[v_{(a)(b)} v_{(a)(b)}-e^{-4 D} R^{(3)}(\widetilde{\mathbf{e}})\right], \\
W_{\text {potential }}=\int d^{4} x N[\underbrace{\frac{4}{3} e^{-7 D / 2} \triangle^{(3)} e^{-D / 2}}_{\text {Newtonian potentials }}]
\end{gathered}
$$

where all definitions are given in the Appendix A devoted to the Dirac Hamiltonian approach to the GR in terms of the tetrades (see Eqs. (A.1) - A.10). 
In particular, $N_{0}$ is the collective lapse function (27), $v_{(a)(b)}$ are given by Eq. (A.6), and the three-dimensional curvature $R^{(3)}(\widetilde{\mathbf{e}})$ is defined by Eq. (A.7).

The action (36) and its representation with the aid of two last terms $W_{\text {graviton }}$ and $W_{\text {potential }}$ is well known. We just reformulated the action in terms of conformal and affine variables (given below in a definite Dirac-ADM frame: $4 \rightarrow 3+1$ (16) and (17) and $3 \rightarrow 2+1$ (68), (69), and (70)). The term $W_{\text {Universe }}$ was suggested in Ref. [15] due to the separation of the dilaton zeroth mode. Here we introduce a new term, $\rho_{\tau}^{\mathrm{v}}$ as a vacuum graviton energy contribution (and other contributions from fields if they are taken into account). The effect of this new term will be discussed below. In Appendix $\mathrm{C}$ we show also that this term allows to obtain a good description of supernovae data developed earlier in the conformal cosmological model [13, 14. In the later one the contribution of the auxiliary scalar field was exploited instead of $\rho_{\tau}^{\mathrm{v}}$. Strong gravitational waves within our model will be discussed in Section 3 .

The introduction of the finite volume $V_{0}=\int_{V_{0}} d^{3} x<\infty$ in $W_{\text {Universe }}$ creates a dimensional parameter, and therefore, it breaks the conformal symmetry. According to the general wisdom [12, the symmetry breaking leads to appearance of a Goldstone mode [32,34. It is just the zeroth harmonic $\langle D\rangle$. Note, however, that the Hamiltonian dynamics governed by the equations of motion must obey the conformal symmetry (see below).

Thus, the action (36), complemented by the condition (34) and field-space evolution generator (33), provides the framework of the Hamiltonian dynamics in terms of the variables (16), (17). This dynamics enables one to determine the perturbation series $\mathcal{N}=1+\bar{\delta} \ldots$ with the consistent constraint $\int d^{3} x \bar{\delta}=0$ in the frame of reference co-moving with the local volume element according to the constraint (34) (see Appendix B).

\subsection{The empty Universe limit}

At the beginning of Universe, in the limit of the tremendous values of the $z$-factor $(a \rightarrow 0)$, the action $W_{\text {Universe }}$ dominates. Therefore, it is natural to neglect the last two terms in Eq. (36), i.e., we consider an empty space.

Recall that in our approach there are two independent variables: the dilaton zeroth mode $\langle D\rangle$ and the global lapse function $N_{0}$. The variation of action (37) with respect to the dilaton zeroth mode leads to the equation of motion:

$$
\frac{\delta W_{\text {Universe }}}{\delta\langle D\rangle}=0 \Rightarrow 2 \partial_{\tau}\left[\partial_{\tau}\langle D\rangle\right]=\frac{d \rho_{\tau}^{\mathrm{v}}}{d\langle D\rangle} .
$$

The variation with respect to the global lapse function leads to the energy constraint

$$
\frac{\delta W_{\text {Universe }}}{\delta N_{0}}=0 \Rightarrow\left[\partial_{\tau}\langle D\rangle\right]^{2}=\rho_{\tau}^{\mathrm{v}} .
$$

This constraint preserves the conformal symmetry of equation of motion (40) with respect to transformations $\langle D\rangle \rightarrow\langle D\rangle+C$, if

$$
\rho_{\tau}^{\mathrm{v}} \equiv H_{\tau}^{2}=H_{0}^{2}=\text { Const. }
$$


The solution of Eqs. (41), (42), determines Eq.(25) in terms of the dilaton time

$$
\langle D\rangle \equiv \ln (1+z) \equiv-\ln a=H_{0} \tau,
$$

which describes the evolution of the redshift with respect to the dilaton time interval $d \tau$.

Note that our equations (40), (41) do not differ from the original Friedmann's ones written in terms of conformal coordinates and observable quantities for a rigid state. Indeed, taking into account Eqs. (28), (43), one finds that Eq.(41) has the rigid state form in terms of the conformal variables (see also Appendix C)

$$
\left[\partial_{\eta} a\right]^{2}=\rho_{\mathrm{cr}} / a^{2},
$$

where

$$
\rho_{\text {cr }}=H_{0}^{2} \frac{3 M_{\mathrm{Pl}}^{2}}{8 \pi}=H_{0}^{2}
$$

is the critical density. This equation leads to the definition of the rigid state horizon

$$
d_{\mathrm{hor}}(a)=2 \int_{a_{I} \rightarrow 0}^{a} d \bar{a} \frac{\bar{a}}{\sqrt{\rho_{\mathrm{cr}}}}=\frac{a^{2}}{H_{0}} .
$$

The evolution of the cosmological scale factor in terms of the conformal timeinterval given by Eqs. (28), (44) yields the coordinate distance - redshift relation for the photon at its light cone $d s_{\mathrm{C}}^{2}=d \eta^{2}-d r^{2}=0$

$$
e^{-\langle D\rangle}=a(\eta)=\sqrt{1+2 H_{0}\left(\eta-\eta_{0}\right)} ; \quad r=\eta-\eta_{0},
$$

as the solution of Eqs. (41) and (42) in terms of the conformal variables (28). It coincides with the Friedmann solution of his equation (C.2) for the dominant rigid state. Here $\eta$ is the instant of the photon emission by a cosmic atom and $\eta_{0}$ is the time of the photon detection at the Earth. In the CGR, the cosmological scale factor (47) provides the cosmic evolution of atomic masses $m(\eta)=a(\eta) m_{0}$ which gives the redshift of the cosmic atom spectrum lines: the far is an atom, the more is its redshift. Therefore, the redshift is produced by the ratio $\lambda(\eta) / \lambda\left(\eta_{0}\right)$, where $\lambda(\eta)$ is the photon wave length of the photon emitted by cosmic atom with the mass $m(\eta)=a(\eta) m_{0}$ and detected at the Earth, where an etalon atom at the Earth has the mass $m_{0}$.

If a measurable photon time is identified with the conformal one, the square root of the conformal time in Eq.(47) means that the Universe was in the $1 / a^{2}$ regime (44) in the epoch of the chemical evolution. The estimation of the primordial helium abundance [35,36] takes into account the square root dependence of the $z$-factor on the measurable time-interval $(1+z)^{-1} \sim \sqrt{t_{\text {measurable }}}$. In the standard cosmology, where the measurable time-interval is identified with the Friedmann time, this square root dependence of the $z$-factor is explained by the radiation dominance. In the conformal cosmology, where the measurable time-interval is identified with the conformal time, the square root 
dependence of the $z$-factor is explained by the universal rigid state $(1+z)^{-1}=$ $a_{I} \sqrt{1+2 H_{I}\left(\eta-\eta_{I}\right)}[36$.

Thus, we found that the empty Universe evolves in time as a rigid state. Below we demonstrate that the same $1 / a^{2}$ dependence is also a feature of the Casimir vacuum energy.

\subsection{Conformal Casimir energy and the Universe horizon}

Let us again consider the Early Universe. We assume that at the instant of creation the world was empty and finite in size. Therefore, its energy can be associated with the quantum Casimir energy of all physical fields in the given space. We shall treat all these fields as massless since $m(a) \stackrel{a \rightarrow 0}{\longrightarrow} 0$ in the Early Universe epoch.

The Casimir energy of a massless field $f$

$$
\mathrm{H}_{\mathrm{Cas}}^{(f)}=\sum_{\mathbf{k}} \frac{\sqrt{\mathbf{k}}^{2}}{2}=\frac{\widetilde{\gamma}^{(f)}}{d_{\mathrm{Cas}}(a)} .
$$

depends on the geometry, size $d_{\text {Cas }}$, topology, boundary conditions, and spin (in particular, for a sphere of diameter $d_{\text {Cas }}$ the number of $\widetilde{\gamma} \sim 0.1 \div 0.03$ ) [32, 37. For simplicity we assume that the Universe has a spherical volume limited by the horizon.

It is natural to suggest that the energy of a massless field is proportional to the inverse visual size of the Universe $d_{\text {Cas }}(a)$. Assuming the same dependence for all fields, we define the total Casimir energy density of the Universe summing over all fields

$$
\rho_{\eta}^{\mathrm{v}}(a)=\sum_{f} \frac{\mathrm{H}_{\mathrm{Cas}}^{(f)}}{V_{0}}=\frac{C_{0}}{d_{\text {Cas }}(a)} .
$$

The key assumption of our model is that the Casimir dimension $d_{\mathrm{Cas}}(a)$ is equal to the Universe visual size (its horizon (C.3))

$$
d_{\text {Cas }}(a) \equiv d_{\text {hor }}(a)=2 \int_{a_{I} \rightarrow 0}^{a} d \bar{a}\left[\rho_{\eta}^{\mathrm{v}}(\bar{a})\right]^{-1 / 2}=2 C_{0}{ }^{-1 / 2} \int_{a_{I} \rightarrow 0}^{a} d \bar{a} d_{\text {Cas }}^{1 / 2} .
$$

Eq.(150) has the solution

$$
d_{\text {Cas }}^{1 / 2}(a)=\left[C_{0}\right]^{-1 / 2} a \rightarrow d_{\text {Cas }}(a)=\frac{a^{2}}{C_{0}} .
$$

Comparing Eqs. (46), (51), one obtains

$$
C_{0}=H_{0}
$$

Thus, in our approach, the parameter $C_{0}$ is equal to the Hubble parameter $H_{0}$ which can be determined from observations. Neglecting all matter effects, 
we obtain a new simple cosmological model. Below we will show that the dominance of the rigid state can persist even after an intensive creation of primordial particles.

\subsection{Hierarchy of cosmological scales}

In this Section we employ the Planck least action postulate to the empty Universe action; define the initial value of the cosmological scale factor, and consider a hierarchy of cosmological scales in correspondence with their conformal weights.

Let us consider the Early Universe at the rigid state horizon (46). A hypothetical observer measures the conformal horizon $d_{\text {hor }}=2 r_{\text {hor }}(z)$ as the distance that a photon covers within its light cone. The latter is determined by the zero interval equation $d \eta^{2}-d r^{2}=0$ during the photon lifetime in the homogeneous Universe, which is subject to the condition $\eta_{\text {hor }}=r_{\text {hor }}(z)=$ $1 /\left[2 H_{0}(1+z)^{2}\right]$, in accordance with Eqs. (43) and (51). This means that the four-dimensional space-time volume restricted by the horizon is equal to

$$
V_{\text {hor }}^{(4)}=\frac{4 \pi}{3} r_{\text {hor }}^{3}(z) \cdot \eta_{\text {hor }}(z)=\frac{4 \pi}{3 \cdot 16 H_{0}^{4}(1+z)^{8}} .
$$

It is natural to assume that at the instant of the Universe origin the world was essentially quantum. In this case, the Universe action can differ from the zero classical one by the least action (or quanta), which presumably be small and be governed by the Planck postulate of the least action for quantum systems. Therefore, we suppose that action (37) is subjected to the Planck's least action postulate at $a_{\mathrm{Pl}}=\left(1+z_{\mathrm{Pl}}\right)^{-1}$

$$
W_{\text {Universe }}=\rho_{\mathrm{cr}} V_{\mathrm{hor}}^{(4)}\left(a_{\mathrm{Pl}}\right)=\frac{M_{\mathrm{Pl}}^{2}}{H_{0}^{2}} \frac{1}{32\left(1+z_{\mathrm{Pl}}\right)^{8}}=2 \pi \text {. }
$$

Using the present day $\left(\tau=\tau_{0}\right)$ observational data for the Planck mass and the Hubble parameter at $h \simeq 0.7$ [3]

$$
\begin{gathered}
M_{\mathrm{C}} e^{\langle D\rangle\left(\tau_{0}\right)}=M_{\mathrm{Pl}}=1.2211 \cdot 10^{19} \mathrm{GeV}, \quad\langle D\rangle\left(\tau_{0}\right)=0, \\
\frac{d}{d \tau}\langle D\rangle\left(\tau_{0}\right)=H_{0}=2.1332 \cdot 10^{-42} \mathrm{GeV} \cdot h=1.4332 \cdot 10^{-42} \mathrm{GeV},
\end{gathered}
$$

we obtain from (54) the primordial redshift value

$$
a_{\mathrm{Pl}}^{-1}=\left(1+z_{\mathrm{Pl}}\right) \approx\left[M_{\mathrm{Pl}} / H_{0}\right]^{1 / 4}[4 / \pi]^{1 / 8} / 2 \simeq 0.85 \times 10^{15} .
$$

In other words, the Plank mass and the present day Hubble parameter value are related to each other by the age of the Universe expressed in terms of the cosmological scale factor.

In field theories characteristic scales, associated with physical states, are classified according to the Poincaré group representation 2,38. In our approach the Poincaré classification of energies arises from the decomposition of 
Table 1 The hierarchy law of the cosmological scales in $\operatorname{GeV}\left(M_{\mathrm{Pl}}^{*}=\sqrt{3 /(8 \pi)} M_{\mathrm{Pl}}\right)$.

\begin{tabular}{|c|c|c|c|c|c|}
\hline $\mathrm{n}$ & $\mathrm{n}=0$ & $\mathrm{n}=1$ & $\mathrm{n}=2$ & $\mathrm{n}=3$ & $\mathrm{n}=4$ \\
\hline$\omega_{0}^{(n)}$ & $H_{0} \simeq 1.4 \cdot 10^{-42}$ & $R_{\mathrm{Cel} . \mathrm{S} .}^{-1} \simeq 1.2 \cdot 10^{-27}$ & $k_{0 \mathrm{CMB}} \simeq 10^{-12}$ & $\phi_{0} \simeq 3 \cdot 10^{2}$ & $M_{\mathrm{Pl}}^{*} \simeq 4 \cdot 10^{18}$ \\
\hline
\end{tabular}

the mean particle energy $\omega_{\tau}=a^{2} \sqrt{\mathbf{k}^{2}+a^{2} M_{0}^{2}}$ conjugated to the dilaton time interval. We express this decomposition in the form

$$
\langle\omega\rangle^{(n)}(a)=\left(a / a_{\mathrm{Pl}}\right)^{(n)} H_{0},
$$

based on Eq. (566.) where $\langle\omega\rangle_{0}^{(0)}=H_{0},\langle\omega\rangle_{0}^{(2)}=k_{0},\langle\omega\rangle_{0}^{(3)}=M_{0},\langle\omega\rangle_{0}^{(4)}=M_{0 \mathrm{Pl}}$.

This equation enables one to introduce the conformal weights $n=0,2,3,4$ which correspond to: the dilaton velocity $v_{D}=H_{0}$, the massless energy $a^{2} \sqrt{\mathbf{k}^{2}}$, the massive one $M_{0} a^{3}$, and the Newtonian coupling constant $M_{\mathrm{Pl}} a^{4}$ (54), respectively. One can also include in this classification the scale of the nonrelativistic particle $H_{0}=a_{\mathrm{Pl}}^{1} \times 10^{-13} \mathrm{~cm}^{-1}$ with the unit conformal weight of its energy $\omega_{\tau}^{\text {nonr. }}=a^{1} \mathbf{k}^{2} / M_{0}$. As a result, the redshift leads to a hierarchy law of the present day $(a=1)$ cosmological scales

$$
\left.\omega_{0}^{(n)} \equiv\langle\omega\rangle^{(n)}(a)\right|_{(a=1)}=\left(1 / a_{\mathrm{Pl}}\right)^{(n)} H_{0}
$$

shown in Table 1 .

Table 1 contains the scales corresponding to the Celestial System size $(n=$ $1)$, the Cosmic Microwave Background mean wave-momentum $(n=2)$, the electroweak scale of the SM $(n=3)$, and the Planck mass $(n=4)$. We conclude that the observational data testify that the cosmic evolution (57) of all these mean energies with conformal weights $(n=0,1,2,3,4)$ has a common origin which could be the Casimir vacuum energy.

Thus, the application of the Planck least action postulate provides the initial value $a_{\mathrm{Pl}}$ given by Eq. (56) in our model. The Poincaré classification of different states, according to their conformal weights, reveals a hierarchy of energy scales in agreement with observations.

\subsection{The exact solution of energy constraint in the CGR}

Let us consider the complete action (36) in variables given by the Dirac-ADM foliation. There are two treatments of the equation $N \delta W / \delta N=0$.

The first one belongs to Arnowitt, Deser and Misner [25], who consider this equation as the definition of the energy component of the total energymomentum tensor related to the Riemannian time $x^{0}$. This treatment leads to the concept of non-localizable energy. However, the latter is not a diffeoinvariant quantity and can not be associated with any observable, since $x^{0}$ is the object of the diffeomorphisms (20). 
The second treatment belongs to Wheeler and DeWitt (WDW) 31, who consider this equation as an algebraic first class energy constraint. Its resolution yields the WDW evolution generator. This generator is identified with the canonical momentum of a time-like variable in the field space.

We conform to the rules of the second route. In particular, in our approach the crucial step is the identification of this diffeo-invariant time-like evolution parameter in the WDW field space-time with the zeroth harmonic of the dilaton field 15. The corresponding canonical momentum is treated as the evolution generator (33) in the Dirac-ADM Hamiltonian approach to the GR. Recall that the zeroth and nonzeroth harmonics of the dilaton field are separated by two projection operators: the "average" $\langle D\rangle$ over the volume and the "deviation" $\bar{D}=D-\langle D\rangle: D=\langle D\rangle+\bar{D}$ defined by Eqs. (22)-(29). This projection removes the interference between the independent degrees of freedom due to the orthogonality condition (24); for example, one has

$$
\frac{1}{V_{0}} \int_{V_{0}} d^{3} x(\langle D\rangle+\bar{D})^{2}=\langle D\rangle^{2}+\frac{1}{V_{0}} \int_{V_{0}} d^{3} x \bar{D}^{2} .
$$

Thus, the GR equations obtained by the variation of action (36) after the separation

$$
N \frac{\delta W_{\mathrm{C}}[D=\langle D\rangle+\bar{D}]}{\delta N}=\left\langle N^{-1}\left[\partial_{0}\langle D\rangle\right]^{2}+N\left[v_{\bar{D}}\right]^{2}\right\rangle
$$

differs from the equations obtained by the variation of this action before the separation

$$
\left.N \frac{\delta W_{\mathrm{C}}[D]}{\delta N}\right|_{D=\langle D\rangle+\bar{D}}=\left.\left\langle N\left[v_{D}\right]^{2}\right\rangle\right|_{D=\langle D\rangle+\bar{D}}
$$

Here $v_{D}$ is given by Eq. A.5 in Appendix A. The logic of the second route requires that in the Hamiltonian approach we have to choose the definite order of operations: the decomposition and the variation of the action.

As a result, the decomposition (22) of the dilaton into two independent harmonics (variables) requires the action to be a function of these two independent variables. The variation of the action with respect to the lapse function $N \frac{\delta W_{\mathrm{C}}}{\delta N}=0$ gives

$$
\frac{\left[\partial_{\tau}\langle D\rangle\right]^{2}-\rho_{\tau}^{\mathrm{v}}}{\mathcal{N}}-\mathcal{N} \widetilde{\mathcal{H}}=0
$$

Here we used Eqs. (26) and (27) to define

$$
\begin{aligned}
& N \frac{\delta}{\delta N} \frac{1}{N_{0}}=N \frac{\delta}{\delta N}\left[\frac{1}{V_{0}} \int_{V_{0}} d^{3} x \frac{1}{N}\right]=-\frac{1}{N}=-\frac{1}{\mathcal{N} N_{0}}, \\
& N \frac{\delta}{\delta N} N_{0}=\frac{N_{0}^{2}}{N}=\frac{N_{0}}{\mathcal{N}}, \quad \frac{[d\langle D\rangle]^{2}}{N_{0}^{2}\left(d x^{0}\right)^{2}}=\left[\partial_{\tau}\langle D\rangle\right]^{2},
\end{aligned}
$$


where we dropped arguments for simplicity. Eq. (62) has the additional term $\left(\left[\partial_{\tau}\langle D\rangle\right]^{2}-\rho_{\tau}^{\mathrm{v}}\right) / \mathcal{N}$ in the comparison with the original Einstein equation $\mathcal{N} \widetilde{\mathcal{H}}=$ 0 [39,40].

Constraint (62) determines the diffeo-invariant lapse function

$$
\mathcal{N}=\frac{\langle\sqrt{\widetilde{\mathcal{H}}}\rangle}{\sqrt{\widetilde{\mathcal{H}}}}
$$

by means of the Hamiltonian density $\widetilde{\mathcal{H}}=-\frac{2}{3} e^{-7 D / 2} \triangle e^{-D / 2}+\mathcal{H}_{g}$ (see Eq. (A.15), A.17) ) and its spatial average $\langle\sqrt{\widetilde{\mathcal{H}}}\rangle=V_{0}^{-1} \int d^{3} x \sqrt{\widetilde{\mathcal{H}}}$. The additional term solves problems of the GR associated with the unambiguous definition of the energy and the lapse function [15. Moreover, it leads also to novel physical consequences for the large-scale structure of the Universe, discussed in detail in the next Section and in Appendix B.

In virtue of this result, by averaging over spatial volume Eq.(62), we obtain the equation for the WDW evolution generator (33):

$$
\left(\partial_{\tau}\langle D\rangle\right)^{2} \equiv \frac{1}{4} P_{\langle D\rangle}^{2}=\rho_{\tau}^{\mathrm{v}}+\mathbf{H}_{\tau}^{g} / V_{0}=\rho_{\text {tot }}^{g} .
$$

Here $\mathbf{H}_{\tau}^{g}$ is the Hamiltonian constructed with the aid of two last terms of action (36).

The solution of Eq. (64) provides the exact time-redshift relation

$$
\tau=\int_{\langle D\rangle_{I}}^{\langle D\rangle_{0}} d\langle D\rangle\left[\rho_{\mathrm{tot}}^{g}\right]^{-1 / 2} .
$$

Thus, the Hamiltonian approach to the CGR provides the exact solution of the energy constraint in terms of the conformal field variables connected with the Einstein ones by the scale transformation

$$
\widetilde{F}^{(n)}=e^{n D} F^{(n)},
$$

where $(n)$ conformal weights $(n=-1,0,3 / 2,2)$ for scalars, vectors, spinors, and tensors, respectively.

The explicit solution of the constraint, Eq. (63), results in the constraintshell interval

$$
\widetilde{d s}^{2}=e^{-4 D} \frac{\langle\sqrt{\mathcal{H}}\rangle^{2}}{\mathcal{H}} d \tau^{2}-\left(\widetilde{\mathbf{e}}_{i(a)} d x^{i}-\overline{\mathcal{N}}_{(a)} d \tau\right)^{2} .
$$

¿From the requirement that the squared time interval is a positive definite it follows that we deal with a field theory with positive-definite metrics of fields in the Hilbert space $\mathcal{H}>0$. This positive-definite metrics is emerged due to condition (34) which is a result of the dilaton decomposition Eq. (22).

The basic cosmological problems are to solve the Hamiltonian equations of motion with respect to the dilaton $\langle D\rangle$ and to establish the relation (65) with observational quantities. 
We stress that the solution (65) of the energy constraint (62) is analogous to the corresponding Einstein equation obtained in the homogeneous approximation. Note that Eq. (63) defines the relation between the lapse function and matter, see Appendix B and Ref. [16].

\section{Affine gravitons and their properties}

\subsection{Affine graviton}

Let us consider the graviton action (38) in order to resolve the constraints arising due to invariance of the action under the general coordinate transformations (21) (i.e. diffeo-invariance).

In the constraint-shell interval (67) only the simplex components $\bar{\omega}_{(a)}(d)=$ $\widetilde{\mathbf{e}}_{i(a)} d x^{i}$ are constrained variables. They obey the condition of the diffeoinvariance. It is one of the main differences of the CGR from the GR.

The choice of the symmetry condition in the CGR leads to the result that follows from the theorem [41]: any arbitrary two-dimensional space metric $d l^{2}=h_{A B} d x^{A} d x^{B},(A, B=1,2)$, can be represented by diffeomorphisms $x^{A} \rightarrow$ $\widetilde{x}^{A}=\widetilde{x}^{A}\left(x^{1}, x^{2}\right)$ in a diagonal form. The result consists in the fact that a kinemetric-invariant nonlinear plane wave moving in the direction $\mathbf{k}$ with the unit determinant $\operatorname{det} h=1$ contains only a single metric component.

In the frame of reference $\mathbf{k}=\left(0,0, k_{3}\right)$ one has $\widetilde{\mathbf{e}}_{(1)}^{1}=e^{g\left(x_{(3)}, \tau\right)}, \widetilde{\mathbf{e}}_{(2)}^{2}=$ $e^{g\left(x_{(3)}, \tau\right)}$, and $\widetilde{\mathbf{e}}_{(3)}^{3}=1$; all other (non-diagonal) components $\widetilde{\mathbf{e}}_{(a)}^{i}$ are equal to zero. Thus, we obtain

$$
\begin{aligned}
& \bar{\omega}_{(1)}=d X_{(1)}-\left[X_{(1)}\right] d g, \\
& \bar{\omega}_{(2)}=d X_{(2)}+\left[X_{(2)}\right] d g, \\
& \bar{\omega}_{(3)}=d x_{3}=d X_{(3)},
\end{aligned}
$$

where a single-component affine graviton $g=g\left(X_{(3)}, \tau\right)$ is a function depending on the time and a single spatial coordinate $X_{(3)}$ in the tangent space $X_{(b)}$. The solutions of the equation $\frac{\delta W}{\delta g}=0 \rightarrow g=g(\eta, X)$ can be expressed via the tangent coordinates:

$$
\begin{gathered}
X_{(1)}=e^{g\left(x_{(3)}, \tau\right)} \bar{x}^{1} \\
X_{(2)}=e^{-g\left(x_{(3)}, \tau\right)} \bar{x}^{2} .
\end{gathered}
$$

Eqs. (68) and (69) mean an expansion (or contraction) of the hypersurface $X_{(A)}(A=1,2)$ perpendicular to the direction of the gravitational wave propagation $X_{(3)}$. A gravitation wave changes the particle velocity via the Hubble like law: the more base, the more additional velocity induced by the graviton.

The exact local Hamiltonian density for the affine graviton is given by (A.17)

$$
\mathcal{H}_{\mathrm{g}}=\left[6 P_{(a)(b)}^{2}+\frac{1}{6} R^{(3)}(\widetilde{\mathbf{e}})\right]
$$


where $R^{(3)}(\mathbf{e})$ and $P_{(a)(b)}^{2}$ are defined by Eqs. (A.8) and A.11), respectively. For the frame of reference $\mathbf{k}=\left(0,0, k_{3}\right)$, we have [22]:

$$
R^{(3)}(\widetilde{\mathbf{e}})=\left(\partial_{(3)} g\right)^{2}, \quad P_{(a)(b)}^{2}=\frac{1}{9}\left[\partial_{\tau} g\right]^{2}
$$

There is a difference of the diffeo-invariant affine graviton from the a metric graviton $g_{i j}^{T T}=g_{j i}^{T T}$ in the GR [22. While the affine graviton has a single degree of freedom, the metric graviton has two traceless and transverse components that satisfy four constraints

$$
\begin{aligned}
& g_{i i}^{T T}=0, \\
& g_{i 3}^{T T}=g_{3 i}^{T T}=0 .
\end{aligned}
$$

In general case of the CGR $\widetilde{\mathbf{e}}_{(b) i}=\mathbf{e}_{(b) i}^{T}$, both the transverse constraint

$$
\partial_{i} \mathbf{e}_{(b) i}^{T}=0
$$

and the unit determinant one

$$
\left|\mathbf{e}_{(b) i}^{T}\right|=1
$$

(as the analog of the Lichnerowicz gauge in the metric formalism 26]) admit to generalize Eqs. (68), (69), and (70) for the linear forms,

$$
\begin{aligned}
\bar{\omega}_{(b)}(d) & =\mathbf{e}_{(b) i}^{T} d x^{i} \\
& =d\left[\mathbf{e}_{(b) i}^{T} x^{i}\right]-x^{j} d \mathbf{e}_{(b) j}^{T} \\
& =d X_{(b)}-X_{(c)} \mathbf{e}_{c}^{T i} d \mathbf{e}_{(b) i}^{T} \\
& =d X_{(b)}-X_{(c)}\left[\omega_{(b)(c)}^{R}+\omega_{(b)(c)}^{L}\right]
\end{aligned}
$$

in the tangent coordinate space. Here $X_{(b)}$ can be obtained by the formal generalization of Eqs. (68), (69), and (70) by means of the Leibniz rule $\mathbf{e}_{(b) i}^{T} d\left[x^{i}\right]=$ $d\left[\mathbf{e}_{(b) i}^{T} x^{i}\right]-x^{i} d \mathbf{e}_{(b) i}^{T}$. The diffeomorphism-invariance admits the choice of the gauge in Eq. (799)

$$
\omega_{(b)(c)}^{L}=0 .
$$

Similar result is valid for a general case of arbitrary wave vector $\mathbf{k}=\frac{2 \pi}{V_{0}^{1 / 3}} \mathbf{l}$, where $X_{(3)}$ is replaced by $X_{(k)}=\mathbf{k X} / \sqrt{\mathbf{k}^{2}}$. The single-component graviton $g(\tau, \mathbf{X})$ considered as the tensor massless representation of the Wigner classification of the Poincaré group [2] can be decomposed into a series of strong waves (in natural units)

$$
\omega_{(a)(b)}^{R}\left(\partial_{(c)}\right)=i \sum_{\mathbf{k}^{2} \neq 0} \frac{e^{i \mathbf{k X}}}{\sqrt{2 \omega_{\mathbf{k}}}} \mathbf{k}_{c}\left[\varepsilon_{(a)(b)}^{R}(k) g_{\mathbf{k}^{+}}^{+}(\eta)+\varepsilon_{(a)(b)}^{R}(-k) g_{-\mathbf{k}^{-}}^{-}(\eta)\right] .
$$


Here $\varepsilon_{(a)(b)}^{R}(\mathbf{k})$ satisfies the constraints

$$
\begin{aligned}
\varepsilon_{(a)(a)}^{R}(k) & =0, \\
\mathbf{k}_{(a)} \varepsilon_{(a)(b)}^{R}(k) & =0,
\end{aligned}
$$

similar to (75), (76)). The variable $\omega_{\mathbf{k}}=\sqrt{\mathbf{k}^{2}}$ is the graviton energy and the affine graviton

$$
\bar{g}_{\mathbf{k}}=\frac{\sqrt{8 \pi}}{M_{\text {Planck }} V_{0}^{1 / 2}} g_{\mathbf{k}}
$$

is normalized to the units of volume and time (like a photon in QED [22]).

In the mean field approximation

$$
\begin{aligned}
\mathcal{N}\left(x^{0}, x^{j}\right) & =1, \quad N^{j}=0, \quad \bar{D}=0, \\
\widetilde{d s}^{2} & =[d \eta]^{2}-\left[\omega_{(b)} \otimes \omega_{(b)}\right],
\end{aligned}
$$

when one neglects all Newtonian-type interactions, the action of an affine graviton reduces to the form of the exact action for the strong gravitational wave 22 .

$$
\begin{aligned}
W_{\text {lin }}^{g} & =\int d \tau \mathbf{L}_{\tau}^{g}, \\
\mathbf{L}_{\tau}^{g} & =\frac{v_{(a)(b)}^{2}-e^{-4 D} R^{(3)}}{6}=\sum_{\mathbf{k}^{2} \neq 0} \frac{v_{\mathbf{k}}^{g} v_{-\mathbf{k}}^{g}-e^{-4 D} \mathbf{k}^{2} \bar{g}_{\mathbf{k}} \bar{g}_{-\mathbf{k}}}{2} \\
& =\left[\sum_{\mathbf{k}^{2} \neq 0} p_{-\mathbf{k}}^{g} v_{\mathbf{k}}^{g}\right]-\mathbf{H}_{\tau}^{g},
\end{aligned}
$$

where $v_{\mathbf{k}}^{g}=\partial_{\tau} \bar{g}_{\mathbf{k}}$ is the derivative with respect to the dilatonic time interval (28) and

$$
\mathbf{H}_{\tau}^{g}=\sum_{\mathbf{k}^{2} \neq 0} \frac{p_{\mathbf{k}^{g}}^{g} p_{-\mathbf{k}}^{g}+e^{-4\langle D\rangle} \mathbf{k}^{2} \bar{g}_{\mathbf{k}} \bar{g}_{-\mathbf{k}}}{2}
$$

is the corresponding Hamiltonian.

Thus, in the mean field approximation (85) the diffeo-invariant sector of the strong gravitational plane waves coincides with a bilinear theory given by Eqs. (87) - 897). In this approximation our model is reduced to a rather simple theory which is bilinear with respect to the single-component graviton field as discussed also in Ref. [16. Note that we consider here the tangential space, and the chosen variables allow to obtain the simple solutions. The main postulated condition here was the requirement of the diffeo-invariance of the graviton equation of motion. While in the standard GR the symmetry properties are required only for the interval, we impose the symmetry with respect to diffeomorphisms also on the Maurer-Cartan forms. 
3.2 Comparison with metric gravitons

It is instructive to compare the properties of the affine and metric gravitons, which was done first in Ref. [16]).

The action of metric gravitons in the accepted GR [42,43, coincides with the affine one (87) in the lowest order of the decomposition over $\mathbf{k}^{2} / M_{\mathrm{Pl}}^{2}$

$$
W_{\text {non-lin }}^{\mathrm{GR}}=W_{\text {lin }}^{g}+W_{\text {non-lin }},
$$

if we keep only diagonal graviton components. It is well-known [33] that the accepted action (90) is highly nonlinear even in the approximation (85).

In the approximation (85), we keep only the dynamical part $\omega_{(c b)}^{R}$ (which enters into the action (87)) and the present day value of the cosmological scale factor $a=e^{-\langle D\rangle}=1$. Let us compare the affine gravitons (79) with the commonly accepted metric gravitons, given by the decomposition [42,43]

$$
\widetilde{d s}_{h}^{2}=(d \eta)^{2}-d x^{i} d x^{j}\left(\delta_{i j}+2 h_{i j}^{T T}+\ldots\right) .
$$

In the accepted case, the graviton moves in the direction of vector $\mathbf{k}$, its wave amplitude $\cos \left\{\omega_{\mathbf{k}} x_{(k)}\right\}$ depends on the scalar product $x_{(k)}=(\mathbf{k} \cdot \mathbf{x}) / \omega_{\mathbf{k}}$.

The graviton changes the squared test particle velocity $\left(\frac{d s}{d \eta}\right)^{2} \sim \frac{d x^{i} d x^{j}}{d \eta d \eta} \varepsilon_{i j}^{\alpha}$ in the plane, orthogonal to the direction of motion. Here $\varepsilon_{i j}^{\alpha}$ is the traceless transverse tensor: $\varepsilon_{i i}^{\alpha}=0$ and $k_{i} \varepsilon_{i j}^{\alpha}=0$. All these effects are produced by the first order of series (91)

$$
\begin{aligned}
d l_{h}^{2} & =2 d x^{i} d x^{j} h_{i j}^{T T}(t, \mathbf{x}) \\
& =d x^{i} d x^{j} \varepsilon_{i j}^{\alpha} \sqrt{6} \cos \left\{\omega_{\mathbf{k}} x_{(k)}\right\}\left(H_{0} / \omega_{\mathbf{k}}\right) \Omega_{\mathbf{k h}}^{1 / 2}+O\left(h^{2}\right),
\end{aligned}
$$

where $H_{0}$ is the Hubble parameter, $\Omega_{\mathbf{k h}}=\omega_{\mathbf{k}} N_{\mathbf{k h}} /\left[V_{0} \rho_{\mathrm{cr}}\right]$ is the energy density of the gravitons in units of the cosmological critical energy density (45). One observes that in the accepted perturbation theory the contribution of a single gravitational wave to the geometrical intervals, Eq. (92), is suppressed by the factor $H_{0} / \omega_{\mathbf{k}}$.

In our version the linear term of the spacial part of Eq. (79) takes the form

$$
d l_{g}^{2}=2 d X_{(b)} X_{(c)} \omega_{(c)(b)}^{R}=d X_{(b)} X_{(c)} \varepsilon_{(c)(b)}^{\alpha} \sqrt{6} \cos \left\{\omega_{\mathbf{k}} X_{(k)}\right\} H_{0} \Omega_{\mathbf{k h}}^{1 / 2} .
$$

Evidently, two models (the GR and the CGR) differ by an additional factor which can be deduced from the ratio

$$
\left|\frac{d l_{h}^{2}}{d l_{g}^{2}}\right|=\left|\frac{d x^{i} d x^{j}\left(h_{i j}^{T T}\right)}{\left(d X_{(b)} X_{(c)} \omega_{(c)(b)}^{R}\right)}\right| \simeq \frac{1}{r_{\perp} \omega_{\mathbf{k}}} \sim \frac{\lambda_{g}}{r_{\perp}} .
$$

Here $r_{\perp}=\sqrt{\left|\mathbf{X}_{\perp}\right|^{2}}$ is the coordinate distance between two test particles in the plane perpendicular to the wave motion direction and $\lambda_{g}$ is the graviton wave length. Therefore, in the CGR there is the effect of the expansion of the plane perpendicular to the affine wave motion direction. 
As a result, in the CGR the total velocity of a test classical particle in the central gravitational field of a mass $M$ and of a strong gravitational wave is the sum of three velocities at the cosmic evolution $a \neq 1$. The first term is the standard Newtonian $(\mathrm{N})$ velocity, the second is the velocity of the graviton expansion $(\mathrm{g})$ in the field of a gravitational wave, and the third one is the velocity of the Hubble evolution $(\mathrm{H})$ :

$$
|\mathbf{v}|^{2}=\left|\frac{d l_{g}}{d \eta}\right|^{2}=[\underbrace{\mathbf{n}_{\mathrm{N}} \sqrt{\frac{r_{g}}{2 R_{\perp}}}}_{\text {Newtonian velocity }}+\underbrace{\mathbf{n}_{\mathrm{g}} \sqrt{R_{\perp} H_{0}} \sqrt{\Omega_{g}}}_{\text {graviton expansion }}+\underbrace{\mathbf{n}_{\mathrm{H}} \gamma H_{0} R_{\perp}}_{\text {Hubble evolution }}]^{2} .
$$

Here, $R_{\perp}=r_{\perp} a(\eta)$ is the Friedman distance from the central mass, $H_{0}$ is the Hubble parameter, $r_{g}\left(R_{\perp}\right)=M / M_{\mathrm{Pl}}^{2}$ is a constant gravitational radius, and

$$
\left\{\begin{array}{l}
\mathbf{n}_{\mathrm{N}}=(0,-1,0) \\
\mathbf{n}_{\mathrm{g}}=(+1 / \sqrt{2},-1 / \sqrt{2}, 0) \\
\mathbf{n}_{\mathrm{H}}=(1,0,0)
\end{array}\right.
$$

are the unit velocity vectors. Their scalar products are $\mathbf{n}_{\mathrm{N}} \cdot \mathbf{n}_{\mathrm{g}} \neq 0, \mathbf{n}_{\mathrm{N}} \cdot \mathbf{n}_{\mathrm{H}}=0$, $\mathbf{n}_{\mathrm{N}} \cdot \mathbf{n}_{\mathrm{g}} \neq 0$, and $\mathbf{n}_{\mathrm{N}} \cdot \mathbf{n}_{\mathrm{H}}=0$. The graviton energy density $\Omega_{\mathrm{g}}$ is given in units of the cosmological critical energy density $\rho_{\text {cr. }}$.

The last two terms provide possible sources of a modified Newtonian dynamics. One observes that the interference of the Newtonian and the gravitoninduced velocities in (94) $v_{\mathrm{n}-\mathrm{g} \text { interf }} \simeq \sqrt[4]{\Omega_{g} r_{g} H_{0}}$ does not depend on the radius $R_{\perp}$.

The third term could imitate the Dark Matter effect in COMA-type clusters with $|R| \sim 10^{25} \mathrm{~cm}$, in accordance with the validity limit of the Newtonian dynamics, $r_{g} / R_{\text {limit }}<2\left(R_{\text {limit }} H_{0}\right)^{2}$, discussed in [44,45]. The factor $\gamma=\sqrt{2}$ is defined by the cosmological density [46].

Thus, in our model strong gravitational waves possess peculiar properties which can be tested by observations and experiments.

\subsection{Vacuum creation of affine gravitons}

Here we are going to study the effect of intensive creation of affine gravitons. We will briefly recapitulate the derivation given in Ref. [16] and further, using the new results of Sect. 2.5, estimate the number of created particles.

The approximation defined by Eqs. (87)- (89) can be rewritten by means of the conformal variables and coordinates, where the action

$$
W_{\text {lin }}^{g}=\int_{\eta_{I}}^{\eta_{0}} d \eta\left[-V_{0}\left(\partial_{\eta}\langle D\rangle\right)^{2} e^{-2\langle D\rangle}+\mathrm{L}_{\eta}^{g}\right]
$$


is given in the interval $\eta_{I} \leq \eta \leq \eta_{0}$ and spatial volume $V_{0}$. Here the Lagrangian and Hamiltonian

$$
\begin{aligned}
\mathbf{L}_{\eta}^{g} & =\sum_{\mathbf{k}^{2} \neq 0} e^{-2\langle D\rangle} \frac{v_{\mathbf{k}}^{g} v_{-\mathbf{k}}^{g}-\mathbf{k}^{2} \bar{g}_{\mathbf{k}} \bar{g}_{-\mathbf{k}}}{2}=\left[\sum_{\mathbf{k}^{2} \neq 0} p_{-\mathbf{k}}^{g} v_{\mathbf{k}}^{g}\right]-\mathrm{H}_{\eta}^{g}, \\
\mathbf{H}_{\eta}^{g} & =\sum_{\mathbf{k}^{2} \neq 0} \frac{e^{2\langle D\rangle} p_{\mathbf{k}}^{g} p_{-\mathbf{k}}^{g}+e^{-2\langle D\rangle} \omega_{0 \mathbf{k}}^{2} \bar{g}_{\mathbf{k}} \bar{g}_{-\mathbf{k}}}{2}
\end{aligned}
$$

are defined in terms of the variables $\bar{g}_{\mathbf{k}}$, their momenta, and one-particle conformal energy

$$
p_{\mathbf{k}}^{g}=e^{-2\langle D\rangle} v_{\mathbf{k}}^{g}=e^{-2\langle D\rangle} \partial_{\eta} \bar{g}_{\mathbf{k}}, \quad \omega_{0 \mathbf{k}}^{g}=\sqrt{\mathbf{k}^{2}},
$$

respectively. The transformation (squeezing)

$$
p_{\mathbf{k}}^{g}=\widetilde{p}_{\mathbf{k}}^{g} e^{-\langle D\rangle}\left[\omega_{0 \mathbf{k}}^{g}\right]^{-1 / 2}, \quad \bar{g}_{\mathbf{k}}=\widetilde{g}_{\mathbf{k}} e^{\langle D\rangle}\left[\omega_{0 \mathbf{k}}^{g}\right]^{1 / 2}
$$

leads to the canonical form

$$
\begin{aligned}
\mathbf{H}_{\eta}^{g} & =\sum_{\mathbf{k}^{2} \neq 0} \omega_{0 \mathbf{k}}^{g} \frac{\widetilde{p}_{\mathbf{k}}^{g} \widetilde{p}_{-\mathbf{k}}^{g}+\widetilde{g}_{\mathbf{k}} \widetilde{g}_{-\mathbf{k}}}{2}=\sum_{\mathbf{k}} \underline{\mathcal{H}}_{\mathbf{k}}^{g}, \\
\underline{\mathcal{H}}_{\mathbf{k}}^{g} & =\frac{\omega_{0 \mathbf{k}}^{g}}{2}\left[\widetilde{g}_{\mathbf{k}}^{+} \widetilde{g}_{-\mathbf{k}}^{-}+\widetilde{g}_{\mathbf{k}}^{-} \widetilde{g}_{-\mathbf{k}}^{+}\right],
\end{aligned}
$$

where

$$
\widetilde{g}_{\mathbf{k}}^{ \pm}=\left[\widetilde{g}_{\mathbf{k}} \mp i \widetilde{p}_{\mathbf{k}}\right] / \sqrt{2}
$$

are the conformal-invariant classical variables in the holomorphic representation 46, 47.

In virtue of Eqs. (99)-(103), the action (96) takes the form

$$
\begin{aligned}
W_{\text {lin }}^{g} & =\int_{\eta_{I}}^{\eta_{0}} d \eta\left[-V_{0}\left(\partial_{\eta}\langle D\rangle\right)^{2} e^{-2\langle D\rangle}-\mathbf{H}_{\eta}^{g}\right] \\
& +\int_{\eta_{I}}^{\eta_{0}} d \eta \sum_{\mathbf{k}^{2} \neq 0} \widetilde{p}_{-\mathbf{k}}\left[\partial_{\eta} \widetilde{g}_{\mathbf{k}}+\partial_{\eta}\langle D\rangle \widetilde{g}_{\mathbf{k}}\right]
\end{aligned}
$$

The evolution equations for this action are

$$
\partial_{\eta} \widetilde{g}_{\mathbf{k}}^{ \pm}= \pm i \omega_{0 \mathbf{k}}^{g} \widetilde{g}_{\mathbf{k}}^{ \pm}+H_{\eta} \widetilde{g}_{\mathbf{k}}^{\mp},
$$

where $H_{\eta}=\partial_{\eta}(\ln a)=-\partial_{\eta}\langle D\rangle$ is the conformal Hubble parameter (in our model $\left.H_{\eta}=H_{0} / a^{2}\right)$. 
It is generally accepted to solve these equations by means of the Bogoliubov transformations

$$
\begin{aligned}
& \widetilde{g}_{\mathbf{k}}^{+}=\alpha_{\mathbf{k}} b_{\mathbf{k}}^{+}+\beta_{-\mathbf{k}}^{*} b_{-\mathbf{k}}^{-}, \\
& \widetilde{g}_{\mathbf{k}}^{-}=\alpha_{\mathbf{k}}^{*} b_{\mathbf{k}}^{-}+\beta_{-\mathbf{k}} b_{-\mathbf{k}}^{+} \text {, } \\
& \alpha_{\mathbf{k}}=\cosh r_{\mathbf{k}}^{g} e^{i \theta_{\mathbf{k}}^{g}}, \quad \beta_{\mathbf{k}}^{*}=\sinh r_{\mathbf{k}}^{g} e^{i \theta_{\mathbf{k}}^{g}},
\end{aligned}
$$

where $r_{\mathbf{k}}^{g}$ and $\theta_{\mathbf{k}}^{g}$ are the squeezing parameter and the rotation one, respectively (see for details reviews [42,32]). These transformations preserve the Heisenberg algebra $O(2 \mid 1)$ [48] and diagonalize Eqs. (105):

$$
\partial_{\eta} b_{\mathbf{k}}^{ \pm}= \pm i \bar{\omega}_{B \mathbf{k}}^{g} b_{\mathbf{k}}^{ \pm}
$$

if the parameters of squeezing $r_{\mathbf{k}}^{g}$ and rotation $\theta_{\mathbf{k}}^{g}$ satisfy the following equations [46]:

$$
\begin{aligned}
\partial_{\eta} r^{g} \mathbf{k} & =H_{\eta} \cos 2 \theta_{\mathbf{k}}^{g}, \\
\omega_{0 \mathbf{k}}^{g}-\partial_{\eta} \theta^{g} \mathbf{k} & =H_{\eta} \operatorname{coth} 2 r_{\mathbf{k}}^{g} \sin 2 \theta_{\mathbf{k}}^{g}, \\
\omega_{B \mathbf{k}}^{g} & =\frac{\omega_{0 \mathbf{k}}^{g}-\partial_{\eta} \theta^{g} \mathbf{k}}{\operatorname{coth} 2 r_{\mathbf{k}}^{g}} .
\end{aligned}
$$

A general solution of the classical equations can be written with the aid of a complete set of the initial data $b_{0 \mathbf{k}}^{ \pm}$:

$$
b_{\mathbf{k}}^{ \pm}(\eta)=\exp \left\{ \pm i \int_{\eta_{0}}^{\eta} d \bar{\eta} \omega_{B \mathbf{k}}^{g}(\bar{\eta})\right\} b_{0 \mathbf{k}}^{ \pm}
$$

On the other hand, quantities $b_{0 \mathbf{k}}^{+}\left(b_{0 \mathbf{k}}^{-}\right)$can be considered as the creation (annihilation) operators, which satisfy the commutation relations:

$$
\left[b_{0 \mathbf{k}}^{-}, b_{0 \mathbf{k}^{\prime}}^{+}\right]=\delta_{\mathbf{k},-\mathbf{k}^{\prime}}, \quad\left[b_{0 \mathbf{k}}^{-}, b_{0 \mathbf{k}^{\prime}}^{-}\right]=0, \quad\left[b_{0 \mathbf{k}}^{+}, b_{0 \mathbf{k}^{\prime}}^{+}\right]=0,
$$

if one introduces the vacuum state as $b_{0 \mathbf{k}}^{-}|0\rangle=0$. Indeed, relations (114) are the results of: i) the classical Poisson bracket $\left\{P_{\widetilde{F}}, \widetilde{F}\right\}=1$ which transforms into

$$
\left[\widetilde{g}_{\mathbf{k}}^{-}, \widetilde{g}_{-\mathbf{k}}^{+}\right]=\delta_{\mathbf{k}, \mathbf{k}^{\prime}}
$$

ii) the solution (113) for the initial data; iii) the Bogoliubov transformations (106), (107).

With the aid of Eqs. (106)-(108) and (113)-(115) we are able to calculate the vacuum expectation value of the total energy (101), (102)

$$
\left\langle 0\left|\mathrm{H}_{\eta}^{g}(a)\right| 0\right\rangle=\sum_{\mathbf{k}} \omega_{0 \mathbf{k}}^{g}\left|\beta_{\mathbf{k}}\right|^{2}=\sum_{\mathbf{k}} \omega_{0 \mathbf{k}}^{g} \frac{\cosh \left\{2 r_{\mathbf{k}}^{g}(a)\right\}-1}{2} .
$$


The numerical analysis [16] of Eqs. (110)-(111) for unknown variables $\left(r_{\mathbf{k}}^{g}, \theta_{\mathbf{k}}^{g}\right)$ with the zero boundary conditions at $a=a_{I}$ (at the beginning of creation)

$$
r_{\mathbf{k}}^{g}\left(a_{I}\right)=0, \quad \theta_{\mathbf{k}}^{g}\left(a_{I}\right)=0
$$

enables us to suggest an approximate analytical solution for the evolution equations.

Our approximation consists in the following. It arises, if instead of $r_{\mathbf{k}}$ one substitutes an approximate value $r_{\text {apr }}$ in the vicinity of the soft mode of the Bogoliubov energy (112) $\omega_{0 \text { appr }}=\partial_{\eta} \theta^{g}$ appr,

$$
\begin{aligned}
& r_{\text {appr }}=\frac{1}{2} \int_{X_{I}=2 \theta_{\mathrm{appr}}^{g}\left(a_{I}\right)}^{X=2 \theta_{\mathrm{appr}}^{g}(a)} \frac{d \bar{X}}{\bar{X}} \cosh \bar{X} \simeq 2\langle D\rangle_{I}, \\
& X(a)=2 \theta_{\mathrm{appr}}^{g}(a)=2 \int_{\eta\left(a_{I}\right)}^{\eta(a)} d \eta \omega_{0 \mathbf{k}} .
\end{aligned}
$$

This soft mode provides a transition [16] at the point $a_{\mathrm{relax}}^{2} \simeq 2 a_{\mathrm{Pl}}^{2}$ from the unstable state of the particle creation to the stable state with almost a constant occupation number during the relaxation time

$$
\eta_{\text {relax }} \simeq 2 e^{-2\langle D\rangle_{I}} /\left(2 H_{0}\right) \equiv 2 a_{I}^{2} /\left(2 H_{0}\right) .
$$

At the point of the relaxation, the determinant of Eqs. (105) changes its sign and becomes positive 49. Finally, we obtain

$$
\left.\left\langle 0\left|\mathcal{H}_{\mathbf{k}}^{g}\right| 0\right\rangle\right|_{\left(a>a_{\text {relax }}\right)}=\omega_{0 \mathbf{k}}^{g} \frac{\cosh \left[2 r_{\mathbf{k}}^{g}\right]-1}{2} \approx \frac{\omega_{0 \mathbf{k}}^{g}}{4 a_{I}^{4}} .
$$

We have verified that the deviation of the results obtained with the aid of this formula from the numerical solutions of Eqs. (110)-(111) (see Ref. [16]) does not exceed $7 \%$.

In virtue of this result, we obtain the total energy

$$
\left.\left\langle 0\left|\mathrm{H}_{\eta}^{g}\right| 0\right\rangle\right|_{\left(a>a_{\text {relax }}\right)} \approx \frac{1}{2 a_{I}^{4}} \sum_{\mathbf{k}} \frac{\omega_{0 \mathbf{k}}^{g}}{2} \equiv \frac{\mathrm{H}_{\eta \mathrm{Cas}}^{g}(a)}{2 a_{I}^{4}},
$$

where $\mathrm{H}_{\eta \text { Cas }}^{g}(a)$ is the Casimir vacuum energy (45) [32,37.

Thus, the total energy of the created gravitons is

$$
\left\langle 0\left|\mathrm{H}_{\eta}^{g}\right| 0\right\rangle \simeq \frac{\widetilde{\gamma} H_{0}}{4 a^{2} a_{I}^{4}}
$$


It appeared that the dilaton initial data $a_{I}=e^{-\langle D\rangle_{I}}$ and $H_{0}$ determine both the total energy (122) of the created gravitons and their occupation number $N_{g}$ at the relaxation time (120):

$$
N_{g}\left(a_{\text {relax }}\right) \simeq \frac{\left\langle 0\left|\mathrm{H}_{\eta}^{g}\right| 0\right\rangle}{\left\langle\omega_{k}^{g}\right\rangle} \simeq \frac{\widetilde{\gamma}^{(g)}}{16 a_{I}^{6}} \simeq 10^{87}
$$

where we divided the total energy by the mean one-particle energy $\left\langle\omega_{k}^{g}\right\rangle \approx$ $\left\langle\omega^{(2)}\right\rangle\left(a_{I}\right)$ defined in Eq. (57). For numerical estimations we use $\widetilde{\gamma}^{(g)} \approx 0.03$. The number of the primordial gravitons is compatible with the number of the CMB photons as it was predicted in Ref. [43.

The main result of this Section consists in the evaluation of the primordial graviton number (124). We suppose that the Casimir energy is defined by the total ground state energy of created excitations, see Eq. (122).

\section{Interaction with fermions}

In this Section, in order to compare our model with the standard approach based on the Einstein's equations, we consider the interaction with matter fields.

Let us consider Einstein's equations

$$
g^{\mu \lambda}\left[R_{\lambda \nu}^{(4)}(g)-\frac{1}{2} g_{\lambda \nu} R^{(4)}(g)\right]=-3 g^{\mu \lambda} T_{\lambda \nu}^{\text {matter }} .
$$

Here

$$
T_{\mu \nu}^{\text {matter }}=-\frac{\left.\delta W_{\text {matter }}\left[g, F^{(n)}\right)\right]}{\delta g_{\mu \nu}}
$$

is the matter energy momentum tensor in the units (15).

These equations are derived by means of the variation of the Hilbert action $\delta W_{\mathrm{H}} / \delta g_{\mu \nu}=0$, where

$$
W_{\mathrm{H}}\left(g, F^{(n)}\right)=\int d^{4} x\left[-\sqrt{-g} \frac{R^{(4)}(g)}{6}\right]+W_{\text {matter }}\left[g, F^{(n)}\right] .
$$

Equations (125) for the metric components $g_{00}$ and $g_{0 j}$ were treated as four first class constraints (in the Dirac definitions [23]). They are consequences of the general coordinate transformations $x \rightarrow \widetilde{x}=\widetilde{x}(x)$ considered as diffeomorphisms.

In order to realize the Weyl's idea of conformal symmetry, Dirac had employed the conformal transformations

$$
\begin{aligned}
g & =e^{-2 D \widetilde{g}}, \\
F^{(n)} & =e^{n D} \widetilde{F}^{(n)}
\end{aligned}
$$

in the Hilbert action with the aid of the scalar dilaton $D$. As a result, he revealed the hidden conformal symmetry of the GR 9 . The identification of the 
new conformal (widetilde) fields with the observational quantities, including the metric components $\widetilde{g}, \widetilde{F}^{(n)}$ is the basic idea of the conformal cosmology [13, 50.51 .

In order to include fermions, we use the Fock simplex in the tetrade formalism [5]:

$$
W_{\text {matter }}[g, \Psi]=\int d^{4} x \sqrt{-g}\left[-\bar{\Psi} i \gamma_{(\beta)} D_{(\beta)} \Psi-m_{0} \bar{\Psi} \Psi\right]
$$

where $\gamma_{(\beta)}=\gamma^{\mu} e_{(\beta) \mu}$ are the Dirac $\gamma$-matrices, summed with tetrades $e_{(\beta) \nu}$, and $m_{0}$ is the present-day fermion mass. The covariant derivative

$$
D_{(\sigma)}=\partial_{(\sigma)}+\frac{i}{2}\left[\gamma_{(\alpha)}, \gamma_{(\beta)}\right] v_{(\alpha)(\beta),(\sigma)}
$$

is given by Eqs. (5) and (6).

Next, we use the Dirac-ADM foliation $(4 \rightarrow 3+1)$ of the tetrades with the lapse function and the shift vector [23. given in Section 2. The Dirac's Hamiltonian approach to the theory begins from the determination of the first class primary constraints. They mean the zero momenta of the time metric components $N, N^{j}$. The first class primary constraints lead to the first class secondary constraints

$$
\begin{aligned}
& P_{N}=\frac{\partial \mathcal{L}_{\mathrm{H}}}{\partial\left(\partial_{0} N\right)}=0 \quad \Rightarrow \quad \frac{\delta W_{\mathrm{H}}}{\delta N}=0, \\
& P_{N^{j}}=\frac{\partial \mathcal{L}_{\mathrm{H}}}{\partial\left(\partial_{0} N^{j}\right)}=0 \quad \Rightarrow \quad \frac{\delta W_{\mathrm{H}}}{\delta N^{j}}=0,
\end{aligned}
$$

where $\mathcal{L}_{\mathrm{H}}$ is the Lagrangian of the Hilbert action $W_{\mathrm{H}}=\int d^{4} x \mathcal{L}_{\mathrm{H}}$. The first class secondary constraints are supplemented by the second class constraints (A.21) and A.22 related to gauge fixing.

The relations between the Conformal and the Standard models can be illustrated using the mass part of the fermion action

$$
W_{\mathrm{m}}[g, \Psi]=-\int d^{4} x \sqrt{-g} m_{0} \bar{\Psi} \Psi,
$$

and the set of its transformations into conformal variables:

$$
\begin{aligned}
& g_{\mu \nu}=e^{-2 D} \widetilde{g}_{\mu \nu}, \quad \Psi=e^{3 D / 2} \widetilde{\Psi} \\
& \sqrt{-\widetilde{g}}=\sqrt{\widetilde{g}_{00}}=e^{-2 D} N, \quad\left|g_{i j}^{(3)}\right|=e^{-3 D} .
\end{aligned}
$$

As a result, we obtain

$$
W_{\mathrm{m}}[N, \widetilde{\Psi}, D]=-\int d^{4} x \sqrt{-\widetilde{g}} m_{0} \overline{\widetilde{\Psi}} \widetilde{\Psi} e^{-D}=-\int d^{4} x N m_{0} \overline{\widetilde{\Psi}} \widetilde{\Psi} e^{-3 D}
$$

One can see that the variations of the action with respect to $N$ and $D$

$$
N \frac{\delta W_{\mathrm{m}}[N, \widetilde{\Psi}, D]}{\delta N}, \quad \frac{\delta W_{\mathrm{m}}[N, \widetilde{\Psi}, D]}{\delta D}
$$


is nothing else but a linear combination of the Einstein's equations (125), i.e. variations of the action (130) in $g$. Thus, the classical tests of general relativity including: perihelion precession of Mercury, deflection of light by the Sun, gravitational redshift of light, and gravitational lensing are completely fulfilled in our case. This correspondence between the GR and its dilatonic version was already discussed by Dirac 9]. Obviously, separation of the dilaton field into zeroth and non-zeroth harmonics suggested in our approach does not change local gravitational interactions with matter, since in the interactions we have always the whole $D=\langle D\rangle+\bar{D}$.

\section{Vacuum creation of Higgs bosons}

In our model the interactions of scalar bosons and gravitons with the dilaton can be treated on the same footing [15]. Using this fact, we would like to consider the intensive creation of the Higgs scalar particles from the vacuum.

To proceed we have to add the SM sector to the theory under construction. In order to preserve the common origin of the conformal symmetry breaking by the Casimir vacuum energy, we have to exclude the unique dimensionful parameter from the SM Lagrangian, i.e. the Higgs term with a negative squared tachyon mass. However, following Kirzhnits [52], we can include the vacuum expectation of the Higgs field $\phi_{0}$, so that: $\phi=\phi_{0}+\bar{h} /[a \sqrt{2}], \int d^{3} x \bar{h}=0$. The origin of this vacuum expectation value $\phi_{0}$ can be associated with the Casimir energy arising as a certain external initial data at $a=a_{\mathrm{Pl}}$. In fact, let us apply the Plank least action postulate to the Standard Model action:

$$
W_{\mathrm{SM}}\left(a_{\mathrm{Pl}}\right) \sim \lambda_{\mathrm{SM}} \phi_{0}^{4} a_{\mathrm{Pl}}^{4} V_{\mathrm{hor}}^{(4)}\left(a_{\mathrm{Pl}}\right)=2 \pi,
$$

where $\lambda_{\mathrm{SM}} \sim 1$ is the Higgs self-coupling and $V_{\mathrm{hor}}^{(4)}\left(a_{\mathrm{Pl}}\right)$ is given by Eq.(153). The relation gives $\phi_{0} \approx a_{\mathrm{Pl}}^{3} H_{0}$, in agreement with its value in Table 1 .

The standard vacuum stability conditions at $a=1$

$$
<0|0>|_{\phi=\phi_{0}}=1,\left.\quad \frac{d<0 \mid 0>}{d \phi}\right|_{\phi=\phi_{0}}=0
$$

yield the following constraints on the Coleman-Weinberg effective potential of the Higgs field:

$$
V_{\text {eff }}\left(\phi_{0}\right)=0, \quad \frac{d V_{\text {eff }}\left(\phi_{0}\right)}{d \phi_{0}}=0 .
$$

It results in a zero contribution of the Higgs field vacuum expectation into the Universe energy density. In other words, the SM mechanism of a mass generation can be completely repeated in the framework of our approach to the spontaneous symmetry breaking.

In particular, one obtains that the Higgs boson mass is determined from the equation $V_{\mathrm{eff}}^{\prime \prime}(\langle\phi\rangle)=M_{h}^{2}$. Note that in our construction the Universe evolution is provided by the dilaton, without making use of any special potential and/or 
any inflaton field. In this case we have no reason to spoil the renormalizablity of the SM by introducing the non-minimal interaction between the Higgs boson and the gravity $[53]$.

In the approximation (85) of theory (36) supplemented by the Standard Model the Higgs bosons are described by the action

$$
W_{h}=\int d \tau \sum_{\mathbf{k}^{2} \neq 0} \frac{v_{\mathbf{k}}^{h} v_{-\mathbf{k}}^{h}-h_{\mathbf{k}}{ }_{-\mathbf{k}} a^{2} \omega_{0 \mathbf{k}}^{h}}{2}=\sum_{\mathbf{k}^{2} \neq 0} p_{-\mathbf{k}}^{h} v_{\mathbf{k}}^{h}-\mathbf{H}_{\tau}^{h},
$$

where

$$
\omega_{0 \mathbf{k}}^{h}(a)=\sqrt{\mathbf{k}^{2}+a^{2} M_{0 \mathrm{~h}}^{2}}
$$

is the massive one-particle energy with respect to the conformal time interval.

There are values of the scale factor $a$, when the mass term in the oneparticle energy is less than the conformal Hubble parameter value $a M_{0 \mathrm{~h}}<$ $H_{0} a^{-2}$. As a result, the Casimir energy for the Higgs particles coincides with the graviton one at the considered epoch:

$$
\mathrm{H}_{\mathrm{Cas}}^{h} \simeq \sum_{\mathbf{k}} \frac{\sqrt{\mathbf{k}^{\mathbf{2}}}}{2}=\mathrm{H}_{\mathrm{Cas}}^{g}
$$

In this case the calculation of the scalar particle creation energy completely repeats the scheme for the graviton creation, discussed above.

Assuming thermalization in the primordial epoch, we expect that the occupation number of the primordial Higgs bosons is of the order of the known CMB photon one

$$
\mathrm{N}_{h} \sim \mathrm{N}_{\gamma}=411 \mathrm{~cm}^{-3} \cdot \frac{4 \pi r_{h}^{3}}{3} \simeq 10^{87}
$$

We point out that this number is of the order of (124). Thus, the CGR provides a finite occupation number of the produced primordial particles. Note that in other approaches 32 a subtraction is used to achieve a finite result. Moreover, the number of produced particles happens to be of the order of the known CMB photon number. To our opinion this coincidence supports our model, since the number of photons can naturally inherit the number of primordial Higgs bosons (if one considers the photons as one of the final decay products of the bosons). According to our model, the relativistic matter has been created very soon after the Planck epoch at $z_{\mathrm{Pl}} \simeq 10^{15}$. Later on it cooled down and at $z_{\mathrm{CMB}} \simeq 1000$ the $\mathrm{CMB}$ photons decoupled from recombined ions and electrons as discussed by Gamow. In our model the CMB temperature is defined directly from the Hubble parameter and the Planck mass (related to the Universe age $\left.a_{\mathrm{Pl}}\right)$. It is a result of the continuous cooling of the primordial relativistic matter till the present day described by Eq. (57). 
Note that the obtained occupation number (143) corresponds to the thermalized system of photons with the mean wave length $\lambda_{\mathrm{CMB}}$ (at the temperature $T \simeq 3^{\circ} \mathrm{K}$ ) in the finite volume $V_{0} \sim H_{0}^{-3}$ :

$$
\left(\mathrm{N}_{\gamma}\right)^{1 / 3} \simeq 10^{29} \simeq \lambda_{\mathrm{CMB}} H_{0}^{-1}
$$

As concerns vacuum creation of spinor and vector SM particles, it is known 32 to be suppressed very much with respect to the one of scalars and gravitons.

\section{Summary}

We developed a Hamiltonian approach to the gravitational model, formulated as the nonlinear realization of joint affine and conformal symmetries proposed long ago in $8,9,6,11$. With the aid of the Dirac-ADM foliation, the conformal and affine symmetries provide a natural separation of the dilaton and gravitational dynamics in terms of the Maurer-Cartan forms. As the result, the exact solution, Eqs. (63) - 65), of the energy constraint yields the diffeo-invariant evolution operator in the field space.

In the CGR, the conformal symmetry breaking happens due to the Casimir vacuum energy (48)-(50). This energy is obtained as a result of the quantization scheme of the Hamiltonian dynamics proposed in Sec. 3. In our approach, the Casimir vacuum energy provides a good description of SNe Ia data [54 in the conformal cosmology 13,14$]$. The diffeo-invariant dynamics in terms of the Maurer-Cartan forms with application of the affine symmetry condition leads to the reduction of the graviton representation to the one-component field. The affine graviton strong wave yields the effect of expansion (or contraction) in the hypersurface perpendicular to the direction of the wave propagation. We demonstrated that the Planck least action postulate applied to the Universe limited by its horizon provides the value of the cosmological scale factor at the Planck epoch. A hierarchy of cosmological energy scales for the states with different conformal weights is found. The intensive creation of primordial gravitons and Higgs bosons is described assuming that the Casimir vacuum energy is the source of this process. We have calculated the total energy of the created particles, Eq. (122), and their occupation numbers, Eqs. (124) and (143).

The presented model is under development. To completely establish or discard it, one has to consider various other problems, including the quantization of the gravitational field, the CMB power spectrum anisotropy, baryon asymmetry, thermalization of primordial particles etc. Evidently, these problems require a dedicated studies and are left for the future.

Acknowledgements The authors would like thank M. Bordag, S. Deser, D. Ebert, A. Efremov, V. Gershun, Yu. Ignatev, E. Lukierski, and A. Zheltukhin for useful discussions. ABA is

\footnotetext{
3 In these papers the rigid state was associated with a homogeneous kinetic energy of a free scalar field.
} 
grateful to the Dynasty foundation. VNP and AB were supported in part by the BogoliubovInfeld program. AFZ is grateful to the JINR Directorate for a support.

\section{A Dirac Hamiltonian Dynamics in Terms of the Maurer-Cartan Forms}

For the sake of comparison of our approach with the standard Dirac one we reformulate the latter in terms of the Maurer-Cartan forms. In order have a more general consideration, we include in the action an electromagnetic field $F_{\mu \nu}=\partial_{\mu} A_{\nu}-\partial_{\nu} A_{\mu}$ and a scalar field $Q$

$$
\begin{aligned}
\widetilde{W}[g, A, Q] & =-\int d^{4} x \sqrt{-g}\left(\frac{1}{6} R^{(4)}(g)-\frac{1}{4} F_{\mu \alpha} F_{\nu \beta} g^{\mu \nu} g^{\alpha \beta}\right. \\
& \left.+\partial_{\mu} Q \partial_{\nu} Q g^{\mu \nu}\right) .
\end{aligned}
$$

Remind that we use the natural units

$$
\hbar=c=M_{\text {Planck }} \sqrt{3 /(8 \pi)}=1 .
$$

With the aid of the definition of the tetrade components Eqs. (9), we obtain the action A.1)

$$
\widetilde{W}=\int d^{4} x N\left[\mathcal{L}_{D}+\mathcal{L}_{g}+\mathcal{L}_{A}+\mathcal{L}_{Q}\right]
$$

Here, the Lagrangian densities are

$$
\begin{aligned}
\mathcal{L}_{D} & =-v_{D}^{2}-\frac{4}{3} e^{-7 D / 2} \triangle e^{-D / 2} \\
\mathcal{L}_{g} & =\frac{1}{6}\left[v_{(a)(b)} v_{(a)(b)}-e^{-4 D} R^{(3)}(\mathbf{e})\right], \\
\mathcal{L}_{A} & =\frac{1}{2}\left[e^{2 D} v_{(b)(\mathrm{A})}^{2}-e^{-2 D} F_{i j} F^{i j}\right], \\
\mathcal{L}_{Q} & =e^{2 D}\left(v_{Q}+v_{D} \widetilde{Q}\right)^{2}-e^{-2 D}\left(\partial_{(b)} \widetilde{Q}+\partial_{(b)} D \widetilde{Q}\right)^{2}
\end{aligned}
$$

and

$$
\begin{aligned}
v_{Q} & =\frac{1}{N}\left[\left(\partial_{0}-N^{l} \partial_{l}\right) \widetilde{Q}+\partial_{l} N^{l} / 3\right] \\
v_{D} & =\frac{1}{N}\left[\left(\partial_{0}-N^{l} \partial_{l}\right) D+\partial_{l} N^{l} / 3\right], \\
v_{(a)(b)} & =\frac{1}{N}\left[\omega_{(a)(b)}^{R}\left(\partial_{0}-N^{l} \partial_{l}\right)+\partial_{(a)} N_{(b)}^{\perp}+\partial_{(b)} N_{(b)}^{\perp}\right], \\
v_{(b)(\mathrm{A})} & =\frac{1}{N} \mathbf{e}_{(a)}^{i}\left[\partial_{0} A_{i}-\partial_{i} A_{0}+F_{i j} N^{j}\right]
\end{aligned}
$$

are velocities of the metric components and fields, $\triangle=\partial_{i}\left[\mathbf{e}_{(a)}^{i} \mathbf{e}_{(a)}^{j} \partial_{j}\right]$ is the BeltramiLaplace operator, and $R^{(3)}(\mathbf{e})$ is a three-dimensional spatial curvature expressed in terms 
of triads $\mathbf{e}_{(a) i}$ (for the sake of discussion we use $\widetilde{\mathbf{e}} \rightarrow \mathbf{e}$ in Appendix A),

$$
\begin{aligned}
R^{(3)} & =R^{(3)}(\mathbf{e})-\frac{4}{3} e^{7 D / 2} \triangle e^{-D / 2}, \\
R^{(3)}(\mathbf{e}) & =-2 \partial_{i}\left[\mathbf{e}_{(b)}^{i} \sigma_{(c) \mid(b)(c)}\right]-\sigma_{(c) \mid(b)(c)} \sigma_{(a) \mid(b)(a)} \\
& +\sigma_{(c) \mid(d)(f)} \sigma_{(f) \mid(d)(c)}, \\
\sigma_{(c) \mid(a)(b)} & =\left[\omega_{(a)(b)}^{L}\left(\partial_{(c)}\right)+\omega_{(a)(c)}^{R}\left(\partial_{(b))}-\omega_{(b)(c)}^{R}\left(\partial_{(a)}\right)\right],\right. \\
\omega_{(a)(b)}^{R}\left(\partial_{(c)}\right) & =\frac{1}{2}\left[\mathbf{e}_{(a)}^{j} \partial_{(c)} \mathbf{e}_{(b)}^{j}+\mathbf{e}_{(b)}^{i} \partial_{(c)} \mathbf{e}_{(a)}^{i}\right], \\
\omega_{(a)(b)}^{L}\left(\partial_{(c)}\right) & =\frac{1}{2}\left[\mathbf{e}_{(a)}^{j} \partial_{(c)} \mathbf{e}_{(b)}^{j}-\mathbf{e}_{(b)}^{i} \partial_{(c)} \mathbf{e}_{(a)}^{i}\right],
\end{aligned}
$$

where $\triangle=\partial_{i}\left[\mathbf{e}_{(a)}^{i} \mathbf{e}_{(a)}^{j} \partial_{j}\right]$ is the Beltrami-Laplace operator.

With help of the Legendre transformations $v^{2} / N=p v-N p^{2} / 4$ we determine momenta

$$
\begin{aligned}
P_{(a)(b)} & =\frac{v_{(a)(b)}}{3}, \\
P_{D} & =2 v_{D}, \\
P_{Q} & =2 v_{Q}, \\
P_{\mathrm{A}(\mathrm{b})} & =v_{\mathrm{A}(\mathrm{b})} .
\end{aligned}
$$

Consequently, the total action A.3 is

$$
\begin{aligned}
\widetilde{W} & =\int d^{4} x\left[P_{Q}\left(\partial_{0} \widetilde{Q}+\partial_{0} D \widetilde{Q}\right)+P_{(a)(b)} \omega_{(a)(b)}^{R}\left(\partial_{0}\right)\right. \\
& \left.+P_{A(b)} \partial_{0} A_{(b)}-P_{D} \partial_{0} D-\mathcal{C}\right], \\
\mathcal{C} & =N \mathcal{H}+N_{(b)} T_{(b)}+A_{(0)} \partial_{(b)} P_{A(b)}+\lambda_{(0)} P_{D} \\
& +\lambda_{(b)} \partial_{k} \mathbf{e}_{(b)}^{k}+\lambda_{A} \partial_{(b)} A_{(b)},
\end{aligned}
$$

where $N, N_{(b)}$ and $A_{(0)} \partial_{(b)}$ with $\partial_{(b)} A_{(b)}=0$ are the Lagrange multipliers of the first class constraints, $\lambda_{(0)}, \lambda_{(b)}$ and $\lambda_{A}$ are the multipliers for the second class ones;

$$
\begin{aligned}
\mathcal{H} & =-\frac{\delta \widetilde{W}}{\delta N}=\mathcal{H}_{D}+\mathcal{H}_{g}+\mathcal{H}_{A}+\mathcal{H}_{Q}, \\
\mathcal{H}_{D} & =-\frac{P_{D}^{2}}{4}-\frac{4}{3} e^{-7 D / 2} \triangle e^{-D / 2}, \\
\mathcal{H}_{g} & =\left[6 P_{(a)(b)}^{2}+\frac{e^{-4 D}}{6} R^{(3)}(\mathbf{e})\right], \\
\mathcal{H}_{A} & =\frac{e^{-2 D}}{2}\left[P_{i(\mathrm{~A})} P_{(\mathrm{A})}^{i}+F_{i j} F^{i j}\right], \\
\mathcal{H}_{Q} & =e^{-2 D}\left[\frac{P_{Q}^{2}}{4}+\left(\partial_{(b)} Q+\partial_{(b)} D Q\right)^{2}\right], \\
T_{(0)(a)} & =-\mathbf{e}_{(b)}^{i} \frac{\delta W}{\delta N_{i}}=-\partial_{(b)} P_{(b)(a)}+\widetilde{T}_{(0)(a)},
\end{aligned}
$$

and $\widetilde{T}_{(0)(a)}=\sum_{F=\bar{\phi}, \bar{Q}, \widetilde{F}} P_{F} \partial_{(a)} F$ are the energy-momentum tensor components. Dirac added the second class (gauge) constraints [23:

$$
\partial_{k} \mathbf{e}_{(b)}^{k}=0,
$$




$$
P_{D}=0 \quad \rightarrow \quad \partial_{0}\left(e^{-3 D}\right)+\partial_{l}\left(N^{l} e^{-3 D}\right)=0 .
$$

The first three of them fix spatial coordinates 23, and $P_{D}=0$ is known as the minimal surface constraint 33] distinguished by the co-moving frame of reference. Using the decomposition

$$
\begin{aligned}
N_{(b)} & =N_{(b)}^{\|}+N_{(b)}^{\perp}, \\
\partial_{(b)} N_{(b)}^{\|} & =\partial_{j} N^{j}, \\
\partial_{(b)} N_{(b)}^{\perp} & =0 \\
P_{(b)(a)} & =P_{(b)(a)}^{\perp}+\partial_{(a)} f_{(b)}^{\perp}+\partial_{(b)} f_{(a)}^{\perp}
\end{aligned}
$$

and the solution of the constraint A.20, one can represent the squared momentum in Eq. A.17) as

$$
P_{(b)(a)}^{2}=\left(P_{(a)(b)}^{\perp}\right)^{2}+\left[\partial_{(a)} f_{(b)}^{\perp}+\partial_{(b)} f_{(a)}^{\perp}\right]^{2},
$$

where $f_{(a)}^{\perp}$ satisfies the equations

$$
\triangle f_{(a)}^{\perp}=\widetilde{T}_{(0)(a)} .
$$

The second class constraint A.22 leads to one more secondary constraint $\delta W / \delta D=-T_{D}=$ 0 , namely,

$$
\begin{aligned}
\left(\partial_{\tau}\right. & \left.-\mathcal{N}_{(b)} \partial_{(b)}\right) P_{D}=T_{D}, \\
T_{D} & =\frac{4}{3}\left[7 \mathcal{N} e^{-7 D / 2} \triangle e^{-D / 2}+e^{-D / 2} \triangle\left[\mathcal{N} e^{-7 D / 2}\right]\right] \\
& -\partial_{D}\left[\mathcal{H}_{g}+\mathcal{H}_{A}+\mathcal{H}_{Q}\right] .
\end{aligned}
$$

In Ref. 33 the Hamiltonian approach to GR is defined in the class of functions $g_{\mu \nu}\left(x^{0}, \mathbf{x}\right)=$ $\eta_{\mu \nu}+O(1 /|\mathbf{x}|)$, where $\eta_{\mu \nu}=$ Diag : $(1,-1,-1,-1)$. As a result, such a theory excludes cosmological evolution.

However, beginning with the pioneering Friedmann results 29 and continuing with the modern development $3,39 \sqrt[40]{3}$, the cosmological evolution can be incorporated into the gravitational theory with non-flat space-time within the infrared dynamics of the type of the zeroth mode sector $g_{\mu \nu}\left(x^{0}\right) \neq \eta_{\mu \nu}$ (see Appendix C). In the paper we follow this direction.

\section{B Dilaton Cosmological Perturbation Theory}

Recall that in general case the local energy density A.15 is

$$
\widetilde{\mathcal{H}}=-\frac{4}{3} e^{-7 D / 2} \triangle e^{-D / 2}+\sum_{J=0,2,3,4,6} e^{-J D} \mathcal{T}_{J}(\widetilde{F}),
$$

where $\triangle=\partial_{i}\left[\mathbf{e}_{(b)}^{i} \mathbf{e}_{(b)}^{j} \partial_{j}\right]$ is the Beltrami-Laplace operator. The sum is over of the densities of states: rigid $(J=0)$, radiation $(J=2)$, matter $(J=3)$, curvature $(J=4), \Lambda$-type term $(J=6)$, respectively, in terms of the conformal fields

$$
\widetilde{F}^{(n)}=e^{n D} F^{(n)},
$$

where $(n)$ is the conformal weight.

In this case, the equation of the nonzeroth harmonics A.30 takes the form 15

$$
T_{D}-\left\langle T_{D}\right\rangle=0
$$


where

$$
\begin{aligned}
T_{D} & =\frac{2}{3}\left\{7 \mathcal{N} e^{-7 D / 2} \triangle e^{-D / 2}+e^{-D / 2} \triangle\left[\mathcal{N} e^{-7 D / 2}\right]\right\} \\
& +\mathcal{N} \sum_{J=0,2,3,4,6} J e^{-J D} \mathcal{T}_{J} .
\end{aligned}
$$

One can solve all Hamiltonian equations (64), (B.1), and (B.3) to define simplex components

$$
\begin{aligned}
& \widetilde{\omega}_{(0)}=e^{-2 D} \mathcal{N} d \tau, \quad \mathcal{N}=\frac{\langle\sqrt{\widetilde{\mathcal{H}}}\rangle}{\sqrt{\widetilde{\mathcal{H}}}}, \\
& \widetilde{\omega}_{(b)}=d X_{(b)}-X_{(c)} \omega_{(c)(b)}^{R}+\mathcal{N}_{(b)} d \tau .
\end{aligned}
$$

Recall that in the lowest order of perturbation theory with respect to the Newton-type potential $\omega_{(c)(b)}^{R}$ describes the free one-component transverse strong gravitational wave considered in Section 3. The longitudinal component of the shift vector $\mathcal{N}_{(b)}$ is unambiguously determined by the constraint (34) that becomes $\partial_{\eta} e^{-3 \bar{D}}+\partial_{(b)}\left(e^{-3 \bar{D}} \mathcal{N}_{(b)}\right)=0$.

For the small deviations $\mathcal{N} e^{-7 \bar{D} / 2}=1-\nu_{1}$ and $e^{-\bar{D} / 2}=1+\mu_{1}+\ldots$ the first orders of Eqs. (B.1) and (B.4) take the form

$$
\begin{aligned}
& {\left[-\hat{\triangle}+14 \rho_{(0)}-\rho_{(1)}\right] \mu_{1}+2 \rho_{(0)} \nu_{1}=\overline{\mathcal{T}}_{(0)},} \\
& {\left[7 \cdot 14 \rho_{(0)}-14 \rho_{(1)}+\rho_{(2)}\right] \mu_{1}+\left[-\hat{\triangle}+14 \rho_{(0)}-\rho_{(1)}\right] \nu_{1}=7 \overline{\mathcal{T}}_{(0)}-\overline{\mathcal{T}}_{(1)},}
\end{aligned}
$$

where

$$
\begin{aligned}
& \rho_{(n)}=\left\langle\mathcal{T}_{(n)}\right\rangle \equiv \sum_{J=0,2,3,4,6}(2 J)^{n}(1+z)^{2-J}\left\langle\mathcal{T}_{J}\right\rangle, \\
& \mathcal{T}_{(n)}=\sum_{J=0,2,3,4,6}(2 J)^{n}(1+z)^{2-J} \mathcal{T}_{J} .
\end{aligned}
$$

In the first order of perturbation with respect to the Newton coupling constant, the lapse function and the dilaton take the forms (see also [15])

$$
\begin{gathered}
e^{-\bar{D} / 2}=1+\frac{1}{2} \int d^{3} y\left[G_{(+)}(x, y) \bar{T}_{(+)}^{(D)}(y)+G_{(-)}(x, y) \bar{T}_{(-)}^{(D)}(y)\right], \\
\mathcal{N} e^{-7 \bar{D} / 2}=1-\frac{1}{2} \int d^{3} y\left[G_{(+)}(x, y) \bar{T}_{(+)}^{(N)}(y)+G_{(-)}(x, y) \bar{T}_{(-)}^{(N)}(y)\right],
\end{gathered}
$$

where $G_{( \pm)}(x, y)$ are the Green functions satisfying the equations

$$
\left[ \pm m_{( \pm)}^{2}-\triangle\right] G_{( \pm)}(x, y)=\delta^{3}(x-y) .
$$

Here

$$
\begin{aligned}
m_{( \pm)}^{2} & =H_{0}^{2} \frac{3(1+z)^{2}}{4}\left[14(\beta \pm 1) \Omega_{(0)}(a) \mp \Omega_{(1)}(a)\right], \\
\beta & =\sqrt{1+\left[\Omega_{(2)}(a)-14 \Omega_{(1)}(a)\right] /\left[98 \Omega_{(0)}(a)\right]}, \\
\bar{T}_{( \pm)}^{(D)} & =\overline{\mathcal{T}}_{(0)} \mp 7 \beta\left[7 \overline{\mathcal{T}}_{(0)}-\overline{\mathcal{T}}_{(1)}\right], \\
\bar{T}_{( \pm)}^{(N)} & =\left[7 \overline{\mathcal{T}}_{(0)}-\overline{\mathcal{T}}_{(1)}\right] \pm(14 \beta)^{-1} \overline{\mathcal{T}}_{(0)},
\end{aligned}
$$


are the local currents, and

$$
\Omega_{(n)}(a)=\sum_{J=0,2,3,4,6}(2 J)^{n}(1+z)^{2-J} \Omega_{J}
$$

$\Omega_{J=0,2,3,4,6}=\left\langle\mathcal{T}_{J}\right\rangle / H_{0}^{2}$ are partial densities of states: rigid, radiation, matter, curvature, $\Lambda$-term, respectively; $\Omega_{(0)}(a=1)=1,1+z=a^{-1}$ and $H_{0}$ is the Hubble parameter.

In the context of these definitions, a full family of solutions (B.10), (B.11) for the lapse function and the nonzeroth dilaton harmonics of the Hamiltonian constraints (62)- 65), yield a Newton-type potential. In particular, for a point mass distribution in a finite volume which corresponds to the nonzero terms with a) $J=0,3$ in Eq.(B.8); b) $J=3$ in Eq.(B.9); c) $J=0,3$ in Eq. B.15 (otherwise zero), - we have

$$
\overline{\mathcal{T}}_{(0)}(x)=\frac{\overline{\mathcal{T}}_{(1)}(x)}{6} \equiv \frac{3}{4 a^{2}} M\left[\delta^{3}(x-y)-\frac{1}{V_{0}}\right] .
$$

As a result, solutions (B.10) and B.11) are transformed to the Schwarzschild-type form

$$
\begin{gathered}
e^{-\bar{D} / 2}=1+\frac{r_{g}}{4 r}\left[\frac{1+7 \beta}{2} e^{-m_{(+)}(a) r}+\frac{1-7 \beta}{2} \cos m_{(-)}(a) r\right], \\
\mathcal{N} e^{-7 \bar{D} / 2}=1-\frac{r_{g}}{4 r}\left[\frac{14 \beta+1}{28 \beta} e^{-m_{(+)}(a) r}+\frac{14 \beta-1}{28 \beta} \cos m_{(-)}(a) r\right],
\end{gathered}
$$

where $r_{g}=M / M_{\mathrm{Pl}}^{2}, \beta=5 / 7, m_{(+)}=3 m_{(-)}$, and $m_{(-)}=H_{0} \sqrt{3(1+z) \Omega_{\mathrm{Matter}} / 2}$. These solutions describe the Jeans-like spatial oscillations of the scalar potentials (B.17) and (B.18) even for the case of zero pressure.

These spatial oscillations can determine the clustering of matter in the recombination epoch, when the redshift is close to the value $z_{\text {recomb. }} \simeq 1100$. Indeed, if we use for the matter clustering parameter (that follows from spatial oscillations of the modified Newton law (B.17), (B.18) ) the observational value 55.

$$
r_{\text {clustering }} \simeq 130 \mathrm{Mpc} \simeq \frac{1}{m_{(-)}}=\frac{1}{H_{0}\left[\Omega_{\mathrm{Matter}}\left(1+z_{\mathrm{recomb}}\right)\right]^{1 / 2}} .
$$

one obtains $\Omega_{\text {Matter }} \sim 0.2$. This estimation is in an agreement with the one, recently discovered in the quest of the large scale periodicity distribution (see for details in [14]).

Constraint (34) yields the shift of the coordinate origin in the process of the evolution

$$
\mathcal{N}^{i}=\left(\frac{x^{i}}{r}\right)\left(\frac{\partial_{\eta} V}{\partial_{r} V}\right), \quad V(\eta, r)=\int^{r} \widetilde{d r} \widetilde{r}^{2} e^{-3 \bar{D}(\eta, \widetilde{r})}
$$

In the limit $H_{0}=0$ at $a_{0}=1$, the solutions (B.17) and (B.18) coincide with the isotropic Schwarzschild solutions: $e^{-\bar{D} / 2}=1+r_{g} /(4 r), \quad \mathcal{N} e^{-7 \bar{D} / 2}=1-r_{g} /(4 r), \quad \mathcal{N}_{i}=0$. Solution B.17) doubles the angle of the photon beam deflection by the Sun field. Thus, the CGR provides also the Newtonian limit in our variables.

\section{Conformal Cosmology}

The distant supernovae data provide a powerful test for all theoretical cosmological models in spite of the fact that the correctness of the hypothesis about $\mathrm{SNe}$ Ia as the perfect standard candles is still not proven [56. However, the first observational conclusion about 
accelerating expansion of the Universe and about the existence of the non-vanishing $\Lambda$-term was made with the cosmological SNe Ia data.

Among different theories that passed this test, see e.g. 50 57, there are conformal cosmological models [13, 36 58, 15 14] which assume to explain the long distance SNe Ia by the long dilaton intervals of the Dirac version of GR [23] considered in the present paper. This type of cosmological model naturally emerges from our approach to the GR, which is based on the conformal symmetry. In this case the unknown dark energy of $\Lambda$-term is replaced by the well known Casimir vacuum energy of the empty Universe.

The construction of all observable CC-quantities is based on the conformal postulate in accord to which each CC-quantity $F_{c}^{(n)}$ with conformal weight $(n)$ is equal to the SC one, $F_{s}^{(n)}$, multiplied by the cosmological scale factor to the power $(-n)$ :

$$
F_{c}^{(n)}=a^{-n} F_{s}^{(n)} .
$$

In accord with the conformal postulate C.1 , the CC-time is greater than the $\mathrm{SC}$ one, and all CC-distances, including the CC-luminosity distance $\ell_{c}$, are longer than the SC-ones $\ell_{s}=a \ell_{c}$, because all intervals are measured by clocks of mass Const/a.

The first attempts to analyze SNe Ia data to evaluate parameters of CC models were made in [13, where only 42 high redshift type Ia SNe [54] point were used. Later a slightly extended sample was analyzed [36. In spite of a small size of the samples used in previous attempts to fit CC model parameters, it was concluded that if $\Omega_{\text {Rigid }}$ is significant with respect to the critical density (45), CC models could fit SNe Ia observational data with a reasonable accuracy. After that a possibility to fit observational SNe Ia data with CC models was seriously discussed by different authors [50,59] among other alternatives.

In both the cosmological models, the dependence of the scale factor $(a)$ on the conformal time $(\eta)$ is given by the Einstein - Friedmann equation 29.

$$
\begin{aligned}
\left(\frac{d a}{d \eta}\right)^{2} & =\rho_{\eta}=H_{0}^{2} \Omega(a), \\
\Omega(a) & \equiv \Omega_{\Lambda} a^{4}+\Omega_{\text {Matter }} a+\Omega_{\text {Radiation }}+\Omega_{\text {Rigid }} a^{-2},
\end{aligned}
$$

where $\Omega(a)$ is the sum of the partial densities: $\Lambda$-term-state, matter, radiation, and rigid, respectively, normalized by the unit density $\left.\Omega\right|_{a=1}=1 ; H_{0}$ is the present-day value of the Hubble parameter. One obtains from Eq. C.2 the definition of the horizon

$$
d_{\mathrm{hor}}(a)=2 r_{\mathrm{hor}}(z)=2 \int_{a_{I} \rightarrow 0}^{a} d \bar{a} \frac{1}{\sqrt{\rho_{\eta}(\bar{a})}}
$$

Thus, this distance determines the diameter of the visible Universe "sphere".

The best fit to the Supernova data 54 requires a cosmological constant $\Omega_{\text {Rigid }}=0$, $\Omega_{\Lambda}=0.7$ and $\Omega_{\text {Matter }}=0.3$ in the $\Lambda \mathrm{CDM}$ model, where the measurable distance is identified with the world space interval $R=a r$.

In the conformal cosmology [13, measurable time and distance are identified with the conformal quantities $(r, \eta)$. Therefore, in the CC framework, we have a possibility to consider the Early Universe evolution 60 using the parameters of the CC dark energy obtained from the SNe Ia data [54. In our CC model, the dark energy is the integral of motion $\rho_{I \text { Cas }} \simeq \rho_{0 \text { Cas }}$ and has the substantial foundation as experimental fact.

\section{References}

1. S. L. Glashow, Partial Symmetries of Weak Interactions, Nucl. Phys. 22, 579 (1961); S. Weinberg, A Model of Leptons, Phys. Rev. Lett. 19, 1264 (1967);

A. Salam, The standard model, Almqvist and Wikdells, Stockholm (1969), in Elementary Particle Theory, ed. N. Svartholm, p.367. 
2. N. N. Bogoliubov, A. A. Logunov, A. I. Oksak, and I. T. Todorov, General Principles of Quantum Field Theory, Springer (1989).

3. M. Giovannini, Theoretical tools for the physics of $C M B$ anisotropies, Int. J. Mod. Phys. D 14 (2005) 363 arXiv:astro-ph/0412601.

4. V. Mukhanov, Physical Foundations of Cosmology, Cambridge University Press, Cambridge (2005);

D. S. Gorbunov and V. A. Rubakov, Introduction to the Theory of the Early Universe: Hot Big Bang Theory, World Scientific, (2010).

5. V. Fock, Geometrization of Dirac's theory of the electron, (in German) Z. Phys. 57, $261(1929)$.

6. V. I. Ogievetsky, Infinite-dimensional algebra of general covariance group as the closure of finite-dimensional algebras of conformal and linear groups, Lett. Nuovo Cim. 8, 988 (1973).

7. A. Einstein, Approximative Integration of the Field Equations of Gravitation, Sitzungsber. Preuss. Akad. Wiss. Berlin (Math. Phys. ) 1916 (1916) 688;

A. Einstein, Über Gravitationswellen (in German) ibid 1918, 154 (1918).

8. S. Deser, Scale invariance and gravitational coupling, Annals Phys. 59, 248 (1970).

9. P. A. M. Dirac, Long range forces and broken symmetries, Proc. Roy. Soc. Lond. A 333, 403 (1973).

10. D. Hilbert, Nachrichten von der Kön. Ges. der Wissenschaften zu Göttingen, (in German) Math.-Phys. Kl., 3, 395 (1915).

11. A. B. Borisov and V. I. Ogievetsky, Theory of Dynamical Affine and Conformal Symmetries as Gravity Theory, Theor. Math. Phys. 21, 1179 (1975) [Teor. Mat. Fiz. 21, 329 (1974)].

12. S. R. Coleman, J. Wess and B. Zumino, Structure of phenomenological Lagrangians. 1, Phys. Rev. 177, 2239 (1969);

D. V. Volkov, Phenomenological Lagrangians, Fiz. Elem. Chast. Atom. Yadra 4, 3 (1973) [Preprint ITF-69-73, Kiev, 1969].

13. D. Behnke, D. B. Blaschke, V. N. Pervushin and D. Proskurin, Description of supernova data in conformal cosmology without cosmological constant, Phys. Lett. B 530, 20 (2002) arXiv:gr-qc/0102039.

14. A. F. Zakharov and V. N. Pervushin, Conformal Cosmological Model Parameters with Distant SNe Ia Data: 'gold' and 'silver', Int. J. Mod. Phys. D 19, 1875 (2010) arXiv:1006.4745 [gr-qc]].

15. B. M. Barbashov, V. N. Pervushin, A. F. Zakharov and V. A. Zinchuk, Hamiltonian cosmological perturbation theory, Phys. Lett. B 633, 458 (2006) arXiv:hep-th/0501242.

16. A. B. Arbuzov, B. M. Barbashov, R. G. Nazmitdinov, V. N. Pervushin, A. Borowiec, K. N. Pichugin and A. F. Zakharov, Conformal Hamiltonian Dynamics of General Relativity, Phys. Lett. B 691, 230 (2010) arXiv:1007.0293 [gr-qc]].

17. D. Blas, M. Shaposhnikov, D. Zenhausern, Scale-invariant alternatives to general relativity, Phys. Rev. D 84, 044001 (2011) arXiv:1104.1392 [hep-th]].

18. J. Garcia-Bellido, J. Rubio, M. Shaposhnikov and D. Zenhausern, Phys. Rev. D 84, 123504 (2011) arXiv:1107.2163 [hep-ph]].

19. R. Penrose, and M. A. H. MacCallum, Twistor Theory: An Approach to the Quantization of Fields and Space-Time, Phys. Rept., 6C, 241-316 (1972).

20. E. Cartan, Lecons sur la geometric des espaces de Riemann, Gauthier-Villars, Paris, (1946).

21. V. N. Pervushin. Dynamical Affine Symmetry and Covariant Perturbation Theory for Gravity, Theor.Math.Phys. 27, 330 (1976) [Teor. Mat. Fiz., 27, 16 (1976)].

22. L. D. Landau and E. M. Lifshitz, Classical Theory of Fields, Pergamon Press, New York (1975).

23. P. A. M. Dirac, The theory of gravitation in Hamiltonian form, Proc. Roy. Soc. Lond. A 246,333 (1958);

Fixation of coordinates in the Hamiltonian theory of gravitation, Phys. Rev. 114,924 (1959).

24. H. Weyl, Gravitation und Elektrizität, Sitzungsber. d. Berl. Akad., 465 (1918).

25. R. Arnowitt, S. Deser, and C. W. Misner, The dynamics of general relativity, in L. Witten, Gravitation: An Introduction to Current Research, 227-265, Wiley, New York (1962). 
26. A. Lichnerowicz, L'integration des equations de la gravitation relativiste et le probleme des $n$ corps (in French) Journ. Math. Pures and Appl. B 37, 23 (1944);

J. W. York, Gravitational degrees of freedom and the initial-value problem, Phys. Rev. Lett. 26, 1656 (1971);

K. Kuchar, A Bubble-Time Canonical Formalism for Geometrodynamics, J. Math. Phys. 13, 768 (1972).

27. A. L. Zelmanov, Chronometric invariants and accompanying coordinates in General Relativity, (in Russian) Dokl. AN USSR 107, 315 (1956);

Kinemetric invariants and their relation to the chronometric invariants of Einstein's theory of gravity (in Russian) ibid 209, 822 (1973).

28. A. Einstein, Cosmological Considerations in the General Theory of Relativity, Sitzungsber. Preuss. Akad. Wiss. Berlin (Math. Phys.) 1917, 142 (1917).

29. A. Friedmann, Über die Krümmung des Raumes (in German) Z. Phys. 10, 377 (1922) [Gen. Rel. Grav. 31, 1991 (1999)];

Über die Möglichkeit einer Welt mit konstanter negativer Krümmung des Raumes, (in German) ibid, 21,306 (1924).

30. B. S. DeWitt, Quantum Theory of Gravity. 1. The Canonical Theory, Phys. Rev. 160, 1113 (1967).

31. J. A. Wheeler, in Batelle Rencontres: 1967, Lectures in Mathematics and Physics, edited by DeWitt C. and Wheeler J.A., New York (1968).

32. A. A. Grib, S. G. Mamaev and V. M. Mostepanenko, Quantum Effects in Strong External Fields, Friedmann Laboratory Publishing, St.Petersburg (1994)

33. L. D. Faddeev and V. N. Popov, Covariant quantization of the gravitational field, Sov. Phys. Usp. 16, 777 (1974) [Usp. Fiz. Nauk 111, 427 (1973)].

34. J. Goldstone, Field Theories with Superconductor Solutions, Nuovo Cimento 19, 154 (1961).

35. M. Fukugita, C. J. Hogan and P. J. E. Peebles, The Cosmic baryon budget, Astrophys. J. 503, 518 (1998)

36. D. Behnke, Conformal Cosmology Approach to the Problem of Dark Matter, $\mathrm{PhD}$ Thesis, Rostock Report MPG-VT-UR 248/04 (2004).

37. A. A. Actor, Scalar quantum fields confined by rectangular boundaries, Fortsch. Phys. 43, 141 (1995);

M. Bordag, G. L. Klimchitskaya, U. Mohideen, and V.M. Mostepanenko, Advances in the Casimir Effect, Oxford University Press Inc., New York (2009).

38. E. P. Wigner, On unitary representations of the inhomogeneous Lorentz group, Ann.of Math 40, 149 (1939).

39. E. M. Lifshits, I. M. Khalatnikov, Problems of relativistic cosmology, Sov. Phys. Usp. 6, 495 (1964) [Uspekhi fiz. nauk, 80, 391 (1963)]; Investigations in relativistic cosmology, Adv. Phys. 12,185 (1963);

J. M. Bardeen, Gauge Invariant Cosmological Perturbations, Phys. Rev. D 22, 1882 (1980).

40. V. F. Mukhanov, H. A. Feldman and R. H. Brandenberger, Theory of cosmological perturbations. Part 1. Classical perturbations. Part 2. Quantum theory of perturbations. Part 3. Extensions, Phys. Rept. 215, 203 (1992).

41. K. P. Tod, Three-dimensional Einstein-Weyl geometry. Geometry of low-dimensional manyfolds, Vol. 1, Durham (1989), pp.237-246; London Math. Soc. Lecture Note Ser., 150 Cambridge University Press, Cambridge (1990).

42. L. P. Grishchuk, Gravitational waves in the cosmos and the laboratory, Sov. Phys. Usp. 20, 319 (1977).

43. S. V. Babak and L. P. Grishchuk, The Energy momentum tensor for the gravitational field, Phys. Rev. D 61, 024038 (2000). arXiv:gr-qc/9907027.

44. A. Einstein and E. G. Straus, The influence of the expansion of space on the gravitation fields surrounding the individual stars, Rev. Mod. Phys. 17, 120 (1945).

45. P. Flin, A. A. Gusev, V. N. Pervushin, S. I. Vinitsky and A. G. Zorin, Cold dark matter as cosmic evolution of galaxies in relative units, Astrophysics 47, 242 (2004). arXiv:astro-ph/0301543.

46. A. F. Zakharov, V. A. Zinchuk, and V. N. Pervushin, Tetrad Formalism and Frames of References in General Relativity, Phys. Part. Nucl. 37, 104 (2006). 
47. V. N. Pervushin and V. I. Smirichinski, Bogolyubov quasiparticles in constrained systems, J. Phys. A 32, 6191 (1999). arXiv:hep-th/9902013.

48. J. von Neumann, Die Eindeutigkeit der Schrödingerschen Operatoren, Math. Ann. 104, $570(1931)$;

T. F. Jordan, N. Mukunda, S. V. Pepper, Irreducible representations of generalized oscillator operators, Math. Phys. 4, 1089 (1963).

49. A. Y. Andreev and D. A. Kirzhnits, Tachyons and the instability of physical systems, Phys. Usp. 39, 1071 (1996).

50. A. G. Riess et al. [Supernova Search Team Collaboration], The farthest known supernova: support for an accelerating universe and a glimpse of the epoch of deceleration, Astrophys. J. 560, 49 (2001) arXiv:astro-ph/0104455;

51. S.K. Banerjee, J.V. Narlikar, N.C. Wickramasinghe, F. Hoyle, G. Burbidge, Possible Interpretations of the Magnitude-Redshift Relation for Supernovae of Type 1A, Astrophys. J. 119, 2583 (2000).

52. D. A. Kirzhnits, The hot Universe and the Weiberg model, JETP Lett. 15, 529 (1972).

53. F. L. Bezrukov and M. Shaposhnikov, The Standard Model Higgs boson as the inflaton, Phys. Lett. B 659, 703 (2008).

54. A. G. Riess et al. [Supernova Search Team Collaboration], Observational evidence from supernovae for an accelerating universe and a cosmological constant, Astron. J. 116 1009 (1998) arXiv:astro-ph/9805201;

P. Astier et al. [The SNLS Collaboration], The Supernova legacy survey: Measurement of omega(m), omega(lambda) and $W$ from the first year data set, Astron. Astrophys. 447, 31 (2006) arXiv:astro-ph/0510447.

55. W. J. Cocke, W. G. Tifft, Statistical procedure and the significance of periodicities in double-galaxy redshifts, Astrophys. J., 368, 383 (1991).

56. N. Panagia, High redshift supernovae: Cosmological implications, Nuovo Cim. B 120, 667 (2005) arXiv:astro-ph/0502247.

57. Z. H. Zhu and M. K. Fujimoto, Constraints on Cardassian expansion from distant type Ia supernovae, Astrophys. J. 585, 52 (2003) arXiv:astro-ph/0303021.

58. D. B. Blaschke, S. I. Vinitsky, A. A. Gusev, V. N. Pervushin and D. V. Proskurin, Cosmological production of vector bosons and cosmic microwave background radiation, Phys. Atom. Nucl. 67, 1050 (2004) [Yad. Fiz. 67, 1074 (2004)] arXiv:hep-ph/0504225.

59. M. Tegmark, Measuring the metric: A Parametrized postFriedmanian approach to the cosmic dark energy problem, Phys. Rev. D 66, 103507 (2002) arXiv:astro-ph/0101354; T. Faulkner, M. Tegmark, E. F. Bunn, Y. Mao, Constraining $f(R)$ gravity as a scalartensor theory, Phys. Rev. D 76, 063505 (2007).

60. A. B. Arbuzov, B. M. Barbashov, A. Borowiec, V. N. Pervushin, S. A. Shuvalov, A. F. Zakharov, General Relativity and Standard Model in Scale-Invariant Variables, Grav. Cosmol. 15, 199 (2009). 